\title{
SECOND-ORDER ELLIPTIC OPERATORS AND HEAT KERNELS ON LIE GROUPS
}

\author{
OLA BRATTELI ${ }^{1}$ AND DEREK W. ROBINSON
}

\begin{abstract}
Arendt, Batty, and Robinson proved that each second-order strongly elliptic operator $C$ associated with left translations on the $L_{p}$-spaces of a Lie group $G$ generates an interpolating family of semigroups $T$, whenever the coefficients of $C$ are sufficiently smooth. We establish that $T$ has an integral kernel $K$ satisfying the bounds

$$
a^{\prime} t^{-d / 2} e^{-b^{\prime}\left|g h^{-1}\right|^{2} / t} e^{-\omega^{\prime} t} \leq K_{t}(g ; h) \leq a t^{-d / 2} e^{-b\left|g h^{-1}\right|^{2} / t} e^{\omega t},
$$

where $d$ is the dimension of $G,\left|g h^{-1}\right|$ is the right invariant distance from $h$ to $g$, and $a^{\prime}, b^{\prime}, \omega^{\prime}$, etc. are positive constants. Both bounds are derived by generalization of Nash's arguments for pure second-order operators on $\mathbf{R}^{d}$.
\end{abstract}

\section{INTRODUCTION}

Arendt, Batty, and Robinson [ABR] recently analyzed properties of secondorder strongly elliptic operators with nonconstant coefficients associated with left translations on the $L_{p}$-spaces over a Lie group $G$. Their principal result established that a large class of such operators generate an interpolating family of semigroups on the $L_{p}$-spaces. We explain this result by demonstrating that these semigroups are determined by a universal integral kernel. We then prove that the kernel satisfies the appropriate parabolic equation and in addition has Gaussian upper and lower bounds. But we begin by introducing some basic notation and definitions.

First, if $(X, \mu)$ is a measure space and $L_{p}(X ; \mu)$ are the corresponding $L_{p^{-}}$ spaces, then an interpolating family of semigroups is defined as a family $T=$ $\left\{T^{(p)}\right\}_{1 \leq p \leq \infty}$ of one-parameter semigroups $T^{(p)}$ on $L_{p}$ satisfying the following two properties:

1. (Continuity) $t \mapsto T_{t}^{(p)}$ is strongly continuous if $p \in[1, \infty)$ and weak ${ }^{*}$ continuous if $p=\infty$.

2. (Consistency) $T_{t}^{(p)} \varphi=T_{t}^{(q)} \varphi$ for all $t \geq 0, \varphi \in L_{p} \cap L_{q}$, and $p, q \in$ $[1, \infty]$.

Received by the editors April 14, 1989.

1980 Mathematics Subject Classification (1985 Revision). Primary 22E30, 35J15; Secondary 47D05.

${ }^{1}$ Permanent address: Institute of Mathematics, University of Trondheim, N-7034, Trondheim, Norway. 
Second, if $G$ is a Lie group and $L_{p}=L_{p}(G ; d g)$ are the $L_{p}$-spaces with respect to left-invariant Haar measure $d g$, then $G$ acts isometrically on each $L_{p}$ by left translations,

$$
(L(g) \varphi)(h)=\varphi\left(g^{-1} h\right) .
$$

If $a_{1}, \ldots, a_{d}$ is a basis of the Lie algebra $\mathfrak{g}$ of $G$, we let $A_{1}, \ldots, A_{d}$ denote the generators of the groups $t \mapsto L\left(e^{-t a_{i}}\right)$. (In principle one should make a distinction between the translation groups and their generators on the different spaces but we avoid this notational complication.) The $C^{n}$-subspaces $L_{p ; n}$ of $L_{p}$ are defined by

$$
L_{p ; n}=\bigcap_{1 \leq i_{1}, \ldots, i_{n} \leq d} D\left(A_{i_{1}} \cdots A_{i_{n}}\right),
$$

and the $C^{n}$-norms $\|\cdot\|_{p ; n}$ are defined by $\|\cdot\|_{p ; 0}=\|\cdot\|_{p}$ and

$$
\|\varphi\|_{p ; n}=\sup _{0 \leq i \leq d}\left\|A_{i} \varphi\right\|_{p ; n-1}
$$

for $\varphi \in L_{p ; n}$ and $n \geq 1$, where $A_{0}=I$. It is also convenient to introduce the spaces $L_{\hat{p}}=L_{p}(G ; d \hat{g})$ defined with respect to right-invariant Haar measure $d \hat{g}$. Note that $d \hat{g}=d g \Delta(g)^{-1}$, where the modular function $\Delta$ is an analytic homomorphism of $G$ into $\mathbf{R}_{+}$. Left translations again act on the $L_{\hat{p}}$-spaces but now one has

$$
\|L(g) \varphi\|_{\hat{p}}=\left(\int_{G} d h \Delta(h)^{-1}\left|\varphi\left(g^{-1} h\right)\right|^{p}\right)^{1 / p}=\Delta(g)^{-1 / p}\|\varphi\|_{\hat{p}} .
$$

If $\hat{A}_{1}, \ldots, \hat{A}_{d}$ denote the generators of the groups $t \mapsto L\left(e^{-t a_{i}}\right)$ on the $L_{\hat{p}}$-spaces one again defines

$$
L_{\hat{p} ; n}=\bigcap_{1 \leq i_{1}, \ldots, i_{n} \leq d} D\left(\hat{A}_{i_{1}} \cdots \hat{A}_{i_{n}}\right)
$$

and sets

$$
\|\varphi\|_{\hat{p} ; n}=\sup _{0 \leq i \leq d}\left\|\hat{A}_{i} \varphi\right\|_{p ; n-1}
$$

for $\varphi \in L_{\hat{p} ; n}$ and $n=1,2, \ldots$, where $\|\cdot\|_{\hat{p} ; 0}=\|\cdot\|_{\hat{p}}$ and $\hat{A}_{0}=I$.

Third, let $c_{0}, c_{i} \in L_{\infty}$ and $c_{i j} \in L_{\infty ; 1}$ be real-valued functions for $i, j=$ $1, \ldots, d$. We interpret each such function as a multiplication operator on $L_{p}$ and $L_{\hat{p}}$. Note that if $c \in L_{\infty ; 1}$, then $c L_{p ; 1} \subseteq L_{p ; 1}$ and $A_{i}(c \varphi)=\left(A_{i} c\right) \varphi+$ $c\left(A_{i} \varphi\right)$. Similarly $c L_{\hat{p} ; 1} \subseteq L_{\hat{p} ; 1}$ and $\hat{A}_{i}(c \varphi)=\left(\hat{A}_{i} c\right) \varphi+c\left(\hat{A}_{i} \varphi\right)$. Consequently one can introduce the second-order differential operator

$$
C^{(p)}=-\sum_{i, j=1}^{d} A_{i} c_{i j} A_{j}+\sum_{i=1}^{d} c_{i} A_{i}+c_{0}
$$

with domain $D\left(C^{(p)}\right)=L_{p ; 2}$, and the corresponding operator

$$
\hat{C}^{(p)}=-\sum_{i, j=1}^{d} \hat{A}_{i} c_{i j} \hat{A}_{j}+\sum_{i=1}^{d} c_{i} \hat{A}_{i}+c_{0}
$$


with $D\left(\hat{C}^{(p)}\right)=L_{\hat{p} ; 2}$, for each $p \in[1, \infty]$. It follows from the structure relations of $\mathfrak{g}$ that one may assume that the matrix $c(g)=\left(c_{i j}(g)\right)$ is symmetric for each $g \in G$ and then we define $C^{(p)}$ and $\hat{C}^{(p)}$ to be strongly elliptic if

$$
\lambda_{m}=\inf \left\{\sum_{i, j=1}^{d} c_{i j}(g) \xi_{i} \xi_{j} ; g \in G, \xi \in \mathbf{R}^{d},|\xi|=1\right\}>0,
$$

i.e., if $c(g)$ is uniformly positive definite. Here $|\xi|=\left(\sum_{i=1}^{d} \xi_{i}^{2}\right)^{1 / 2}$. The constant $\lambda_{m}$ is called the ellipticity constant of the operator. In addition it is useful to introduce

$$
\lambda_{M}=\sup \left\{\sum_{i, j=1}^{d} c_{i j}(g) \xi_{i} \xi_{j} ; g \in G, \xi \in \mathbf{R}^{d},|\xi|=1\right\},
$$

the upper ellipticity constant.

The starting point of the present investigation is the following result from [ABR].

Theorem 1.1. Let $d$ be the dimension of $G$. Assume that the operators $C^{(p)}$ and $\hat{C}^{(p)}$ are strongly elliptic. If $c_{i} \in L_{\infty ; n}$ and $c_{i j} \in L_{\infty ; n+1}$ with $n>d / 2$ then there exists an interpolating family $T=\left\{T^{(p)}\right\}_{1 \leq p \leq \infty}$ of semigroups on $L_{p}$ and a second family $\hat{T}=\left\{\hat{T}^{(p)}\right\}_{1 \leq p \leq \infty}$ on $L_{\hat{p}}$ such that the generators of $T^{(p)}$ and $\hat{T}^{(p)}$ are extensions of $C^{(p)}$ and $\hat{C}^{(p)}$, respectively.

The generator of $T^{(2)}$ is $C^{(2)}$, the generator of $\hat{T}^{(2)}$ is $\hat{C}^{(2)}$, and the generator of $T^{(\infty)} \quad\left(=\hat{T}^{(\infty)}\right)$ is the weak ${ }^{*}$-closure of $C^{(\infty)}$.

The families $T$ and $\hat{T}$ are consistent, i.e., $T_{t}^{(p)} \varphi=\hat{T}_{t}^{(q)} \varphi$ for all $t \geq 0$, $\varphi \in L_{p} \cap L_{\hat{q}}$, and $p, q \in[1, \infty]$.

Each of the semigroups $T^{(p)}, \hat{T}^{(q)}$ is positive.

This result is essentially contained in Theorems 3.12 and 3.13 of [ABR]. In fact these theorems are based upon a slightly stronger continuity condition for the coefficients $c_{i}, c_{i j}$ and reach the stronger conclusion that there exists a continuous semigroup $T^{(0)}$ on $C_{0}(G)$ which is consistent with $T$ and $\hat{T}$. Nevertheless the above result follows easily from the proofs of [ABR].

In addition, it follows from [ABR, Theorem 3.13] that $\left\|T_{t}^{(\infty)}\right\|_{\infty \rightarrow \infty} \leq e^{-\omega_{m} t}$, where $\omega_{m}=\inf \left\{c_{0}(g) ; g \in G\right\}$, and $\left\|T_{t}^{(1)}\right\|_{1 \rightarrow 1} \leq e^{-\omega_{1} t}$, where $\omega_{1}=$ $\inf \left\{c_{0}(g)-\sum_{i} A_{i}\left(c_{i}\right)(g) ; g \in G\right\}$. Therefore $\left\|T_{t}^{(p)}\right\|_{p \rightarrow p} \leq e^{-\omega t}$ with $\omega=$ $\omega_{1} \vee \omega_{m}$ independent of $c_{i j}$ and $p$. Similar uniform bounds are valid for $\left\|\hat{T}_{t}^{(p)}\right\|_{\hat{p} \rightarrow \hat{p}}$.

Finally, $T^{(2)}$ is holomorphic and it follows from the Stein interpolation theorem [Ste1 or Ste2], that each $T^{(p)}$ with $p \in\langle 1, \infty\rangle$ is also holomorphic. This general argument does no establish holomorphy of $T^{(1)}$ or $T^{(\infty)}$ although this is to be expected. In fact there should be a common sector of holomorphy for 
all the $T^{(p)}$ determined by the coefficients $c_{i j}$. Again similar properties are valid for the $\hat{T}$.

In the sequel we demonstrate that the semigroups $T^{(p)}$ and $\hat{T}^{(q)}$ are all determined by a universal integral kernel. For this we rely upon the estimation procedures of [Rob].

\section{SEMIGROUP KERNELS}

Let $\mathscr{L}_{\hat{p}}$ denote the closure of the tensor product $L_{\hat{\infty}} \otimes L_{\hat{p}}$ with respect to the norm ||$\cdot \mid \|_{\hat{p}}$, where

$$
\||| f \mid\|_{\hat{p}}=\underset{g \in G}{\operatorname{ess} \sup }\left(\int_{G} d \hat{h}|f(g ; h)|^{p}\right)^{1 / p}
$$

if $p \in[1, \infty\rangle$, and

$$
\||f|\|_{\infty}=\underset{g, h \in G}{\operatorname{ess} \sup |f(g ; h)| .}
$$

Note that we can define an action of left translations on $\mathscr{L}_{\hat{p}}$ by

$$
(L(g) f)(h ; k)=f\left(g^{-1} h ; k\right)
$$

and then extend the definition of $A_{1}, \ldots, A_{d}$ by identifying them with the generators of the groups $t \mapsto L\left(e^{-t a_{i}}\right)$. Since the first variable is related to the space $L_{\infty}=L_{\infty}$, it is also consistent to identify $A_{i}$ with $\hat{A}_{i}$. Then we can define the strongly elliptic operators $C^{(\infty)}=\hat{C}^{(\infty)}$ on each $\mathscr{L}_{p}$ with $D\left(C^{(\infty)}\right)=$ $D\left(\hat{C}^{(\infty)}\right)$ equal to the subspace of the twice left-differentiable elements.

Now we return to the setting of Theorem 1.1.

Theorem 2.1. Suppose that the operators $C^{(p)}$ and $\hat{C}^{(p)}$ are strongly elliptic. Assume that $c_{i} \in L_{\infty ; n}$ and $c_{i j} \in L_{\infty ; n+1}$ with $n>d / 2$. Then for each $t>0$ there is a positive function $K_{t} \in \bigcap_{1 \leq p \leq \infty} \mathscr{L}_{\hat{p}}$ such that

$$
\left(T_{t}^{(p)} \varphi\right)(g)=\int_{G} d \hat{h} K_{t}(g ; h) \varphi(h)
$$

for all $\varphi \in L_{p}, p \in[1, \infty]$, and

$$
\left(\hat{T}_{t}^{(q)} \psi\right)(g)=\int_{G} d \hat{h} K_{t}(g ; h) \psi(h)
$$

for all $\psi \in L_{\hat{q}}, q \in[1, \infty]$.

The kernel $K$ has the following properties:

1. There exist $a, \omega>0$ such that

$$
\left\|\left|K_{t}\right|\right\|_{\hat{p}} \leq a t^{-d\left(1-p^{-1}\right) / 2} e^{\omega t}
$$

for all $t>0$ and $p \in[1, \infty]$. The values of $a$ and $\omega$ depend on the ellipticity constants $\lambda_{m}, \lambda_{M}$ of $c_{i j}$, the norms $\left\|c_{i}\right\|_{\infty ; 1}$ of the $c_{i}$, and $\omega_{m}=\inf \left\{c_{0}(g) ; g \in G\right\}$. 
2. $K_{s+t}(g ; k)=\int_{G} d \hat{h} K_{s}(g ; h) K_{t}(h ; k)$ for all $s, t>0$ and $g, k \in G$.

3. $t \mapsto K_{t}$ is $\|\cdot \cdot\| \|_{p}$-analytic for each $p \in\langle 1, \infty]$.

4. $g \mapsto K_{t}(g ; \cdot)$ is twice left-differentiable with respect to \|\|$\cdot\|\|_{\hat{p}}$ if $p \in$ $[1,2]$. Moreover, $(t, g) \rightarrow K_{t}(g ; h)$ satisfies the parabolic equation

$$
\frac{\partial K_{t}}{\partial t}+C^{(\infty)} K_{t}=0
$$

and $K_{t}(g ; h) \rightarrow \delta\left(g h^{-1}\right)$ weakly as $t \rightarrow 0$.

5. $e^{-\omega_{M} t} \leq \int_{G} d \hat{h} K_{t}(g ; h) \leq e^{-\omega_{m} t}$ for all $t>0$, where $\omega_{M}=\sup \left\{c_{0}(g)\right.$;

$g \in G\}$ and $\omega_{m}=\inf \left\{c_{0}(g) ; g \in G\right\}$.

Before giving the proof we make four remarks about the theorem.

First, suppose that the $T^{(p)}$ have an integral kernel $K$ and the coefficients of the $C^{(p)}$ are constant. Then $T^{(p)}$ commutes with right translations $R$ on $L_{p}$ and it follows from (2.1) that

$$
\begin{gathered}
\int_{G} d \hat{h} K_{t}(g k ; h k) \varphi(h)=\left(R(k) T_{t}^{(p)} R\left(k^{-1}\right) \varphi\right)(g) \\
=\left(T_{t}^{(p)} \varphi\right)(g)=\int_{G} d \hat{h} K_{t}(g ; h) \varphi(h) .
\end{gathered}
$$

Therefore one must have

$$
K_{t}(g ; h)=K_{t}\left(g h^{-1} ; e\right)=K_{t}\left(e ; h g^{-1}\right) .
$$

Hence setting $K_{t}(g)=K_{t}(g ; e)$ one finds

$$
\left(T_{t}^{(p)} \varphi\right)(g)=\int_{G} d \hat{h} K_{t}\left(g h^{-1}\right) \varphi(h)=\int_{G} d h K_{t}(h) \varphi\left(h^{-1} g\right) .
$$

Consequently $T^{(p)}$ is connected with left-invariant Haar measure $d g$ and left translations $L$ through the relation

$$
T_{t}^{(p)}=\int_{G} d h K_{t}(h) L(h) .
$$

This is the convention adopted in [Rob].

Second, if $C^{(p) *}$ is defined on $L_{q ; 2}$ by

$$
C^{(p) *}=-\sum_{i, j=1}^{d} A_{i} c_{i j} A_{j}-\sum_{i=1}^{k} c_{i} A_{i}+c_{0}-\sum_{i=1}^{d} A_{i}\left(c_{i}\right),
$$

then Theorem 1.1 applies to the $C^{(p) *}$ and the corresponding interpolating semigroup $T^{*}$ consists of the adjoints $T^{(p) *}$ of the $T^{(p)}$. Thus if $T$ is determined by the integral kernel $K$ and $T^{*}$ by the kernel $K^{*}$ it follows from the action (2.1) that

$$
K_{t}^{*}(g ; h)=K_{t}(h ; g) \Delta(g)^{-1} \Delta(h) .
$$

Third, the existence of the kernel $K$ is a direct consequence of the estimates of [Rob] and the Dunford-Pettis theorem (see, for example, [Tre or CFKS]) formulated with respect to the $L_{\hat{p}}$-spaces. Specifically we use the following: 
Proposition 2.2. Let $A$ be a bounded operator on $L_{\hat{p}}$, where $p \in[1, \infty\rangle$. The following conditions are equivalent:

1. A is bounded from $L_{\hat{p}}$ to $L_{\hat{\infty}}$.

2. There is an $a \in \mathscr{L}_{\hat{q}}$, where $q$ is the conjugate of $p$, i.e., $p^{-1}+q^{-1}=1$, such that

$$
(A \varphi)(g)=\int_{G} d \hat{h} a(g ; h) \varphi(h) \quad \text { for all } \varphi \in L_{\hat{p}} .
$$

Moreover, if these conditions are satisfied, then $\||a|\|_{\hat{q}}=\|A\|_{\hat{p} \rightarrow \infty}$.

This result shows that the proof of existence of the kernel $K$ is reduced to establishing bounds on the cross-norms $\left\|T_{t}^{(p)}\right\|_{\hat{p} \rightarrow \hat{\infty}}$.

Fourth, the Dunford-Pettis theorem presupposes that $G$ is separable. The connected component $G_{0}$ of the identity element $e \in G$ is separable since $G$ is a Lie group, but $G$ may have uncountably many connected components. This is no great problem, however, as the operators $A$ we will consider generate the semigroups $T_{t}^{(p)}$ and these leave the $L_{\hat{p}}$-spaces over each connected component of $G$ invariant. Thus we may apply the Dunford-Pettis theorem on each component to obtain the kernel $a(g ; h)$ if $g, h$ are in the same component, i.e., if $g h^{-1} \in G_{0}$, while $a(g ; h)=0$ if $g, h$ are in different components. We also note that in proving Theorems $2.1,3.1$, and 4.1 we may assume that $G$ is connected by the following reasoning: If these theorems have been established for $G_{0}$, and $H$ is a connected component of $G$, then there exists an element $k \in G$ such that $H=G_{0} k$.

Now, if we conjugate the original operator

$$
C^{(p)}=-\sum_{i, j=1}^{d} A_{i} c_{i j} A_{j}+\sum_{i=1}^{d} c_{i} A_{i}+c_{0}
$$

with right translation, we obtain an operator $C_{k}^{(p)}$ of the same form, but the new coefficient functions $c_{i j}, c_{i}, c_{0}$ are right translates of the old ones. Since all the derivatives involved in the definitions of the norms $\left\|c_{i j}\right\|_{\infty, 1}$ etc. are left derivatives, the norms associated with the new operator are the same as the old ones. Since the $A_{i}$ commute with right translations, the kernel $K^{k}$ of the semigroups of the translated operators are the right translates of the original kernel:

$$
K_{t}^{k}(g ; h)=K_{t}(g k ; h k) .
$$

But since the theorems have been established for $G_{0}$, one has estimates for $K_{t}^{k}(g ; h)$ in terms of $\left|g h^{-1}\right|=\left|(g k)(h k)^{-1}\right|$ for $g, h \in G_{0}$. Letting $k$ vary over $G$, and using the convention $\left|g h^{-1}\right|=\propto$ if $g h^{-1} \notin G_{0}$, one then obtains corresponding estimates for all $g, h \in G$.

Proof of Theorem 2.1. As a result of the Dunford-Pettis theorem in the form Proposition 2.2, we can simultaneously establish that $K$ exists and satisfies Property 1 of Theorem 2.1 by proving the following lemma. 
Lemma 2.3. There exist $a, \omega>0$ such that

$$
\left\|T_{t}^{(p)}\right\|_{\hat{p} \rightarrow \infty} \leq a t^{-d / 2 p} e^{\omega t}
$$

for all $t>0$ and $p \in[1, \infty]$. The $a$ and $\omega$ depend on the coefficients of $C$ in the manner described in Theorem 2.1.1.

Proof. This lemma extends to general $G$ Nash's first result [Nas] for parabolic equations on $\mathbf{R}^{d}$. The proof follows the arguments developed for higher order elliptic operators with constant coefficients in [Rob]. In particular it uses the general versions of Nash's inequalities, Proposition 3.2 and Corollary 3.4. Similar calculations from different starting points occur in [CKS, Var].

First one derives a bounded on $\left\|\hat{T}_{t}^{(p)}\right\|_{\hat{p} \rightarrow \hat{2}}$ for $p \in[1,2\rangle$ by a differential inequality. If $\varphi \in L_{\hat{2}}$, then

$$
\frac{d}{d t}\left\|\hat{T}_{t}^{(2)} \varphi\right\|_{\hat{2}}^{2}=-\left\langle\hat{C}^{(2)} \hat{T}_{t}^{(2)} \varphi, \hat{T}_{t}^{(2)} \varphi\right\rangle-\left\langle\hat{T}_{t}^{(2)} \varphi, \hat{C}^{(2)} \hat{T}_{t}^{(2)} \varphi\right\rangle
$$

for $t>0$, where $\langle\cdot, \cdot\rangle$ denotes the $L_{\hat{2}}$-scalar product, i.e.,

$$
\langle\varphi, \psi\rangle=\int_{G} d \hat{g} \overline{\varphi(g)} \psi(g) .
$$

Since $\hat{T}^{(2)}$ is holomorphic on $L_{\hat{2}}$ [ABR], one has that $\hat{T}_{t}^{(2)} L_{\hat{2}} \subseteq D\left(\hat{C}^{(2)}\right)=L_{\hat{2} ; 2}$ for all $t>0$ and hence no domain problems occur. Now the generators $\hat{A}_{i}$ of left translations satisfy the adjoint relation

$$
\left\langle\hat{A}_{i} \varphi, \psi\right\rangle=-\left\langle\varphi,\left(\hat{A}_{i}-\beta_{i} I\right) \psi\right\rangle,
$$

where $\beta_{i}=A_{i}(\Delta)(e)$. Therefore if $\psi \in L_{\hat{2} ; 2}$ one has

$$
\begin{aligned}
\left\langle\hat{C}^{(2)} \psi, \psi\right\rangle+\left\langle\psi, \hat{C}^{(2)} \psi\right\rangle= & 2 \sum_{i, j=1}^{d}\left\langle\hat{A}_{i} \psi, c_{i j} \hat{A}_{j} \psi\right\rangle-2 \operatorname{Re} \sum_{i, j=1}^{d}\left\langle\hat{A}_{i} \psi, c_{i j} \psi\right\rangle \beta_{j} \\
& -2 \operatorname{Re} \sum_{i=1}^{d}\left\langle c_{i} \psi, \hat{A}_{i} \psi\right\rangle+2\left\langle\psi, c_{0} \psi\right\rangle .
\end{aligned}
$$

But then it follows from the ellipticity condition on $c_{i j}$ and straightforward estimates that there exist $\lambda>0$ and $\mu \geq 0$ such that

$$
\left\langle\hat{C}^{(2)} \psi, \psi\right\rangle+\left\langle\psi, \hat{C}^{(2)} \psi\right\rangle \geq \lambda \sum_{i=1}^{d}\left\|\hat{A}_{i} \psi\right\|_{\hat{2}}^{2}-\mu\|\psi\|_{\hat{2}}^{2} .
$$

The values of $\lambda$ and $\mu$ depend on the ellipticity constants $\lambda_{m}, \lambda_{M}$ of $c_{i j}$, the norms $\left\|c_{i}\right\|_{\infty}$ of the $c_{i}$, and $\omega_{m}=\inf \left\{c_{0}(g) ; g \in G\right\}$. Consequently one obtains the differential inequality

$$
\frac{d}{d t}\left\|\hat{T}_{t}^{(2)} \varphi\right\|_{\hat{2}}^{2} \leq-\lambda \sum_{i=1}^{d}\left\|\hat{A}_{i} \hat{T}_{t}^{(2)} \varphi\right\|_{\hat{2}}^{2}+\mu\left\|\hat{T}_{t}^{(2)} \varphi\right\|_{\hat{2}}^{2} .
$$


But this implies

$$
\frac{d}{d t}\left\|\hat{T}_{t}^{(2)} \varphi\right\|_{\hat{2}}^{2} \leq-\lambda\left\|\hat{T}_{t}^{(2)} \varphi\right\|_{\hat{2} ; 1}^{2}+(\lambda+\mu)\left\|\hat{T}_{t}^{(2)} \varphi\right\|_{\hat{2}}^{2}
$$

or, equivalently,

$$
\frac{d}{d t}\left(\left\|\hat{T}_{t}^{(2)} \varphi\right\|_{\hat{2}}^{2} e^{-(\lambda+\mu) t}\right) \leq-\lambda\left\|\hat{T}_{t}^{(2)} \varphi\right\|_{\hat{2} ; 1}^{2} e^{-(\lambda+\mu) t} .
$$

Next it follows from Proposition 3.2 of [Rob] with $m=1$ that

$$
\|\varphi\|_{\hat{2}}^{2}=\left\|\Delta^{-1 / 2} \varphi\right\|_{2}^{2} \leq \varepsilon\left\|\Delta^{-1 / 2} \varphi\right\|_{2 ; 1}^{2}+C(\varepsilon c)^{-d \delta}\|\varphi\|_{\hat{r}}^{2}
$$

for all $\varphi \in L_{\hat{r}} \cap L_{\hat{2} ; 1}$, all $\varepsilon>0$, and $r \in[1,2]$, where $\delta=r^{-1}-2^{-1}$. But

$$
\left\|\Delta^{-1 / 2} \varphi\right\|_{2 ; 1}^{2} \leq\|\varphi\|_{\hat{2} ; 1}^{2}+(|\beta| / 2)\|\varphi\|_{\hat{2}}^{2}
$$

where $|\beta|=\sup _{1 \leq i \leq d}\left|\beta_{i}\right|$, and hence

$$
\|\varphi\|_{\hat{2}}^{2} \leq(1-\varepsilon|\beta| / 2)^{-1}\left(\varepsilon\|\varphi\|_{\hat{2} ; 1}^{2}+C(\varepsilon c)^{-d \delta}\|\varphi\|_{\hat{r}}^{2}\right)
$$

whenever $\varepsilon|\beta| / 2<1$. Since $\|\varphi\|_{\hat{2}} \leq\|\varphi\|_{\hat{2} ; 1}$, by definition it follows that one has bounds

$$
\|\varphi\|_{\hat{2}}^{2} \leq \varepsilon\|\varphi\|_{\hat{2} ; 1}^{2}+C^{\prime}(\varepsilon c)^{-d \delta}\|\varphi\|_{\hat{r}}^{2}
$$

for all $\varepsilon>0$ and $\varphi \in L_{\hat{r}} \cap L_{\hat{2} ; 1}$. Next, following Nash, we can minimize the right-hand side with respect to $\varepsilon$ and obtain bounds

$$
\|\varphi\|_{\hat{2}}^{2} \leq k\left(\|\varphi\|_{\hat{2} ; 1}^{2}\right)^{d \delta /(1+d \delta)}\left(\|\varphi\|_{\hat{r}}^{2}\right)^{1 /(1+d \delta)} .
$$

Now (2.4), with $\varphi$ replaced by $\hat{T}_{t}^{(r)} \varphi$, combined with (2.3) leads to a differential inequality

$$
\frac{d}{d t}\left(\left\|\hat{T}_{t}^{(r)} \varphi\right\|_{\hat{2}}^{2} e^{-(\lambda+\mu) t}\right) \leq-k^{\prime}\left(\left\|\hat{T}_{t}^{(r)} \varphi\right\|_{\hat{2}}^{2}\right)^{1+1 / d \delta}\left(\left\|\hat{T}_{t}^{(r)} \varphi\right\|_{\hat{r}}^{2}\right)^{-1 / d \delta} e^{-(\lambda+\mu) t}
$$

where we have used $\hat{T}_{t}^{(r)} \varphi=\hat{T}_{t}^{(2)} \varphi$ for $\varphi \in L_{\hat{2}} \cap L_{\hat{r}}$.

But it follows from the discussion following Theorem 1.1 that one has bounds $\left\|\hat{T}_{t}^{(r)} \varphi\right\|_{\hat{r}} \leq e^{\omega t}\|\varphi\|_{\hat{r}}$, where $\omega$ can be estimated in terms of $\left\|A_{i}\left(c_{i}\right)\right\|_{\infty}$ and $\omega_{m}=\inf \left\{c_{0}(g) ; g \in G\right\}$.

Hence one obtains the final differential inequality

$$
\frac{d}{d t}\left(\left\|\hat{T}_{t}^{(r)} \varphi\right\|_{\hat{2}}^{2} e^{-(\lambda+\mu) t}\right)^{-1 / d \delta} \geq k^{\prime}(d \delta)^{-1}\|\varphi\|_{\hat{r}}^{2} e^{(\lambda+\mu+\omega) t / d \delta} .
$$

Solving this inequality leads to bounds

$$
\left\|\hat{T}_{t}^{(r)}\right\|_{\hat{r} \rightarrow \hat{2}}=\left\|T_{t}^{(r)}\right\|_{\hat{r} \rightarrow \hat{2}} \leq a t^{-d \delta / 2} e^{\omega^{\prime} t}
$$

with $\delta=r^{-1}-2^{-1}, r \in[1,2]$. The values of $a$ and $\omega$ are independent of $r$.

Similar arguments apply to the dual semigroup $T^{*}$ and since $\left\|T_{t}^{(r) *}\right\|_{\hat{r} \rightarrow \hat{2}}=$ $\left\|T_{t}^{(2)}\right\|_{\hat{2} \rightarrow \hat{s}}$, where $s$ is conjugate to $r$, one finds

$$
\left\|T_{t}^{(2)}\right\|_{\hat{2} \rightarrow \hat{s}} \leq a t^{-d\left(2^{-1}-s^{-1}\right) / 2} e^{\omega^{\prime} t}
$$


for all $s \in[2, \infty]$. Therefore,

$$
\left\|T_{t}^{(r)}\right\|_{\hat{r} \rightarrow \hat{s}} \leq a^{\prime} t^{-d\left(r^{-1}-s^{-1}\right) / 2} e^{\omega^{\prime} t}
$$

for a suitable $a^{\prime}>0$ and for $1 \leq r \leq 2 \leq s \leq \infty$. Finally, since one has bounds $\left\|T_{t}^{(p)}\right\|_{\hat{p} \rightarrow \hat{p}} \leq e^{\omega t}$ for all $p \in[1, \infty]$, the statement of Lemma 2.3 follows from the Riesz-Thorin interpolation theorem applied to the scale of spaces $L_{\hat{p}}$.

Proposition 2.2 with $A=T_{t}$ and Lemma 2.3 establish the existence of the kernel $K_{t} \in \bigcap_{1<p \leq \infty} \mathscr{L}_{\hat{p}}$ and the bounds

$$
\left\|\mid K_{t}\right\|_{\hat{p}} \leq a t^{-d\left(1-p^{-1}\right) / 2} e^{\omega t}
$$

for all $p \in\langle 1, \infty]$. But, since the bounds extend by continuity to $p=1$, one also has $K_{t} \in \mathscr{L}_{\hat{1}}$.

Since the kernel $K$ is universal for all $T^{(p)}$ and $\hat{T}^{(q)}$, one can now simplify notation by omitting the suffixes $p$ and $q$. We will make this simplification throughout the sequel.

Property 2 of $K$, the convolution semigroup property, follows immediately from the semigroup property.

In order to prove Property 3 it is convenient to introduce the difference operators $\Delta_{s}, s>0$, by

$$
\Delta_{s} K_{t}=\left(K_{t}-K_{t+s}\right) / s
$$

and the powers $\Delta_{s}^{n}$ by

$$
\Delta_{s}^{n} K_{t}=\left(\Delta_{s}^{n-1} K_{t}-\Delta_{s}^{n-1} K_{s+t}\right) / s .
$$

For analyticity of $t \mapsto K_{t}$ with respect to \|\|$\cdot\|\|_{\hat{p}}$ it then suffices to establish estimates

$$
\left\|\Delta_{s}^{n} K_{t}\right\|_{\hat{p}} \leq b c^{n} n !
$$

for all $n=1,2, \ldots$ and all small $s>0$. But

$$
\begin{aligned}
\left\|\Delta_{s}^{n} K_{t}\right\|_{\hat{p}} & =s^{-n}\left\|\left(I-T_{s}\right)^{n} T_{t}\right\|_{\hat{q} \rightarrow \infty} \\
& \leq s^{-n} \int_{0}^{s} d s_{1} \cdots \int_{0}^{s} d s_{n}\left\|T_{s_{1}+\cdots+s_{n}} C^{n} T_{t}\right\|_{\hat{q} \rightarrow \infty} .
\end{aligned}
$$

Now choose $r \in\langle 1, \infty\rangle$ with $r \geq q$. This is possible because $p>1$ and hence $q<\infty$. Next one has bounds

$$
\left\|T_{s}\right\|_{\hat{q} \rightarrow \hat{q}} \leq e^{\omega^{\prime} s}
$$

by the discussion in $\S 1$,

$$
\left\|C^{n} T_{t / 3}\right\|_{\hat{r} \rightarrow \hat{r}} \leq k^{n} n ! t^{-n} e^{\omega^{\prime} t}
$$

because $T$ is holomorphic on $L_{r}$, and

$$
\left\|T_{t / 3}\right\|_{\hat{q} \rightarrow \hat{r}}\left\|T_{t / 3}\right\|_{\hat{r} \rightarrow \infty} \leq a t^{-d / 2 q} e^{\omega t}
$$


by Lemma 2.3. Therefore

$$
\left\|\mid \Delta_{s}^{n} K_{t}\right\| \|_{\hat{p}} \leq\left(e^{\omega^{\prime} s}\right)^{n}\left(e^{\omega^{\prime} t / 3}\right)\left(k^{n} n ! t^{-n} e^{\omega^{\prime \prime} t}\right)\left(a t^{-d / 2 q} e^{\omega t}\right) .
$$

Hence $t \mapsto K_{t}$ is $\||\cdot|\|_{\hat{p}}$-analytic.

In order to prove that $g \mapsto K_{t}(g ; \cdot)$ is twice-differentiable in the $\left.\||\cdot|\|\right|_{\hat{p}^{-}}$ topology it suffices to prove

$$
\sup _{0<|h| \leq 1}\left\|\left.||(I-L(h))^{2} K_{t}\left|\|_{\hat{p}} /\right| h\right|^{2}<\infty\right.
$$

(see [BR, Proposition 3.1.23]). But

$$
\|\|(I-L(h))^{2} K_{t}\left\|_{\hat{p}}=\right\|(I-L(h))^{2} T_{t} \|_{\hat{q} \rightarrow \hat{\infty}}
$$

and since $p \leq 2$ one has $q \geq 2$. Now if $\varphi \in L_{\hat{q}}$ one has bounds

$$
\left|\left((I-L(h))^{2} T_{t} \varphi\right)(g)\right| \leq c\left\|(I-L(h))^{2} T_{t} \varphi\right\|_{\hat{2} ; n}
$$

by the Sobolev embedding theorem [ABR, Proposition 3.9]. Thus,

$$
\left|\left((I-L(h))^{2} T_{t} \varphi\right)(g)\right| /|h|^{2} \leq c^{\prime}\left\|T_{t} \varphi\right\|_{\hat{2} ; n+2} .
$$

Now, since $c_{i j} \in L_{\infty ; n+1}$ and $c_{i} \in L_{\infty ; n}$, it follows from Corollary 3.8 of [ABR] that

$$
\left\|T_{t} \varphi\right\|_{\hat{2} ; n+2} \leq k\left\|(\lambda I+C)^{n / 2+1} T_{t} \varphi\right\|_{\hat{2}}
$$

for large positive $\lambda$. ([ABR, Corollary 3.8] is in fact formulated for $T$ on $L_{2}$ but it is straightforward to verify a similar result for $T$ on $L_{\hat{2}}$.) Next, since $T$ is holomorphic on $L_{\hat{2}}$, one has bounds

$$
\left\|T_{t} \varphi\right\|_{\hat{2} ; n+2} \leq k^{\prime}\left(1+t^{-n / 2-1}\right) e^{\omega^{\prime} t}\left\|T_{t / 2} \varphi\right\|_{\hat{2}} .
$$

Therefore, by Lemma 2.3 one concludes that

$$
\left\|T_{t} \varphi\right\|_{\hat{2} ; n+2} \leq a^{\prime}\left(1+t^{-n / 2-1}\right) t^{-d\left(2^{-1}-q^{-1}\right) / 2} e^{\omega^{\prime \prime} t}\|\varphi\|_{\hat{q}} .
$$

Combining the various estimates one has

$$
\left\|\left.(I-L(h))^{2} K_{t}\left|\|_{\hat{p}} /\right| h\right|^{2} \leq a^{\prime \prime}\left(1+t^{-n / 2-1}\right) t^{-d\left(2^{-1}-q^{-1}\right) / 2} e^{\omega^{\prime \prime} t}\right.
$$

for $0<|h| \leq 1$. Thus $K$ is twice left-differentiable.

It now follows, however, that $K_{t} \in D(C)$ if $p \in[1,2]$, where the elliptic operator $C$ is defined on $\mathscr{L}_{\hat{p}}$ with the coefficients $c_{i}, c_{i j}$, and the generators of left translations $k \mapsto K_{t}\left(k^{-1} g ; h\right)$. Thus $C=C^{(\infty)} \otimes I$ in tensor product notation. Correspondingly $T$ acts on $\mathscr{L}_{\hat{p}}$ by

$$
\left(T_{s} f\right)(g ; h)=\int_{G} d \hat{k} K_{s}(g ; k) f(k ; h)
$$

for all $f \in \mathscr{L}_{\hat{p}}$. Thus, if $p \in[1,2]$ one calculates that

$$
C K_{t}=\lim _{s \rightarrow 0}\left(I-T_{s}\right) K_{t} / s=\lim _{s \rightarrow 0}\left(K_{t}-K_{s+t}\right) / s=-\partial K_{t} / \partial t,
$$

where the limits exist with respect to the norms $\||\cdot|\|_{\hat{p}}$. 
Finally it follows from Proposition 2.2 applied to $T$ and Proposition 3.12 of $[\mathrm{ABR}]$ that

$$
\int_{G} d \hat{h} K_{t}(g ; h) \leq\left\|T_{t}\right\|_{\hat{\infty} \rightarrow \hat{\infty}} \leq e^{-\omega_{m} t} .
$$

Conversely if $1 \in L_{\infty}$ denotes the identity function, then $\left(C-c_{0}\right) 1=0$. Therefore, the semigroup $T^{\prime}$ generated by $C^{\prime}=C-c_{0}$ must satisfy $T_{t}^{\prime} 1=1$. Hence

$$
\begin{aligned}
T_{t} 1-e^{-\omega_{M} t} 1 & =T_{t} 1-T_{t}^{\prime} 1 e^{-\omega_{M} t} \\
& =\int_{0}^{1} d \lambda T_{\lambda t}\left(\omega_{M}-c_{0}\right) T_{(1-\lambda) t}^{\prime} 1 e^{-\omega_{M} t} \geq 0
\end{aligned}
$$

because $\left(\omega_{m}-c_{0}\right) T_{(1-\lambda) t}^{\prime} 1=\left(\omega_{m}-c_{0}\right) 1 \geq 0$ and $T$ is positive. Consequently

$$
\int_{G} d \hat{h} K_{t}(g ; h)=\left(T_{t} 1\right)(g) \geq e^{-\omega_{M} t} .
$$

This completes the proof of Property 5 and the proof of the theorem.

Although we only proved that $g \mapsto K_{t}(g ; \cdot)$ is twice \|\|$\cdot\|\|_{\hat{p}}$-differentiable for $p \in[1,2]$, it is to be expected that a similar result is true for $p \in\langle 2, \infty\rangle$, at least if the coefficients $c_{i}$ and $c_{i j}$ are sufficiently differentiable. In fact the above argument using the Sobolev lemma shows that the greater the differentiability of the coefficients the greater the differentiability of $K$.

\section{UPPER BOUNDS ON KERNELS}

The semigroup kernels $K$ introduced in $\S 2$ satisfy Gaussian upper and lower bounds. The upper bounds follow by combining the Nash inequality arguments used to prove Lemma 2.3 together with a perturbation argument of Davies [Dav]. If $g \in G$ is the connected component of the unit element $e \in G$, let $|g|$ be the distance from $e$ to $g$ as defined by Garding [Går]. If $g$ is not in the connected component, define $|g|=+\infty$, and adopt the convention $\infty \cdot 0=0$ in Theorem 3.1.

Theorem 3.1. There exist $a, \omega>0$ such that

$$
\left(\int_{G} d \hat{h}\left(e^{\rho\left|g h^{-1}\right|} K_{t}(g ; h)\right)^{p}\right)^{1 / p} \leq a t^{-d\left(1-p^{-1}\right) / 2} e^{\omega\left(1+\rho^{2}\right) t}
$$

for all $g \in G, t>0, \rho \geq 0$, and $p \in[1, \infty)$. Consequently there exist $a, b>0$ and $\omega \geq 0$ such that

$$
0 \leq K_{t}(g ; h) \leq a t^{-d / 2} e^{-b\left|g h^{-1}\right|^{2} / t} e^{\omega t}
$$

for all $g, h \in G$ and $t>0$. The values of $a, b$, and $\omega$ depend on the ellipticity constants $\lambda_{m}, \lambda_{M}$ of $c_{i j}$, the norms $\left\|c_{i}\right\|_{\infty ; 1}$ of $c_{i}$, and $\omega_{m}=\inf \left\{c_{0}(g) ; g \in\right.$ $G\}$, but are independent of $\rho$. (In particular, $K_{t}(g ; h)=0$ if $g$ and $h$ lie in different connected components of $G$.) 
Proof. First, (3.2) follows from (3.1) because in the limit $p \rightarrow \infty$ one obtains the bounds

$$
K_{t}(g ; h) \leq a t^{-d / 2} e^{\omega t} e^{\omega \rho^{2} t} e^{-\rho\left|g h^{-1}\right|}
$$

for all $g, h \in G$ and $\rho, t>0$. Minimizing the right-hand side of this inequality with respect to $\rho$ immediately yields (3.2).

To prove (3.1) we follow an idea of Davies and replace the elliptic operator $C$ by the operator $C_{\rho}=e^{\rho \psi} C e^{-\rho \psi}$, where $\rho \in \mathbf{R}$ and $\psi$ denotes the operator of multiplication by a $C^{\infty}$-function $\psi$. Since

$$
e^{\rho \psi} A_{i} e^{-\rho \psi}=A_{i}-\rho A_{i}(\psi),
$$

the operator $C_{\rho}$ is again of the class considered in [ABR] and, in particular, the $C_{\rho}$ generate an interpolating family of semigroups $T^{\rho}$. Clearly

$$
T_{t}^{\rho}=e^{\rho \psi} T_{t} e^{-\rho \psi}
$$

and hence the kernels $K^{\rho}$ and $K$ of $T^{\rho}$ and $T$ satisfy

$$
K_{t}^{\rho}(g ; h)=c^{\rho(\psi(g)-\psi(h))} K_{t}(g ; h) .
$$

Now Lemma 2.3 applies to $T^{\rho}$ and gives bounds on $\left\|\left|K^{\rho}\right| \mid\right\|_{\hat{q}}$. In fact repetition of the proof of Lemma 2.3 with the explicit form of $C_{\rho}$ gives

$$
\left\|\left|K^{\rho}\right|\right\|_{\hat{q}}=\left\|T_{t}^{\rho}\right\|_{\hat{p} \rightarrow \infty} \leq a t^{-d / 2 p} e^{\omega\left(1+\rho^{2}\right) t},
$$

where $a$ and $\omega$ are independent of $\rho$ and depend on $\psi$ only through the seminorm

$$
N_{2}(\psi)=\sup \left\{\left|\left(A_{i} \psi\right)(g)\right|,\left|\left(A_{i} A_{j} \psi\right)(g)\right| ; g \in G, i, j=1, \ldots, d\right\} .
$$

Explicitly one has bounds

$$
\left(\int_{G} d \hat{h}\left(e^{\rho(\psi(g)-\psi(h))} K_{t}(g ; h)\right)^{q}\right)^{1 / q} \leq a t^{-d / 2 p} e^{\omega\left(1+\rho^{2}\right) t}
$$

for $\rho, t \geq 0$ which are uniform for all $\psi \in C^{\infty}$ with $N_{2}(\psi) \leq 1$. Then it follows by the limiting arguments given in [Rob, §4] that

$$
\left(\int_{G} d \hat{h}\left(e^{\rho\left|g h^{-1}\right|} K_{t}(g ; h)\right)^{q}\right)^{1 / q} \leq a t^{-d / 2 p} e^{\omega\left(1+c \rho^{2}\right) t}
$$

with the value of the additional constant $c$ independent of $\rho$.

Note that we have made no attempt to optimize the values of $b$ and $\omega$. This is possible if one restricts attention to operators of the form

$$
C=-\sum_{i, j=1}^{d} A_{i} c_{i j} A_{j}
$$

but it requires more sophisticated arguments (see, for example, [Var, Dav, FaS]). Typically one establishes that

$$
K_{t}(g ; h) \leq a t^{-d / 2}\left(1+\left|g h^{-1}\right|^{2} / t\right)^{d / 2} e^{-\left|g h^{-1}\right| / 4 \lambda_{M} t},
$$


which is the best possible Gaussian bound for $t$ bounded away from zero. Similar optimal results cannot be expected if one has first order terms. For example, if $G=\mathbf{R}$ and $C=-d^{2} / d x^{2}+c d / d x$, then

$$
\begin{aligned}
K_{t}(x) & =t^{-1 / 2} e^{-(x-c t)^{2} / 4 t}=t^{-1 / 2} e^{-x^{2} / 4 t} e^{-c^{2} t} e^{c x / 2} \\
& \leq t^{-1 / 2} e^{-(1-\varepsilon) x^{2} / 4 t} e^{-(1-1 / 4 \varepsilon) c^{2} t}
\end{aligned}
$$

for all $\varepsilon>0$. Thus optimizing the exponent of the Gaussian term automatically increases the exponent of the $t$-dependent term.

\section{LOWER BOUNDS ON KERNELS}

The semigroup kernels $K$ introduced in $\S 2$ also satisfy a Gaussian lower bound. In this section we combine the upper bound of $\S 3$ with techniques of Fabes and Stroock $[\mathrm{FaS}]$ and Nash [Nas] to establish

Theorem 4.1. There exist positive constants $a, b$, and $\omega$, depending only on $\lambda_{m}, \lambda_{M},\left\|c_{i}\right\|_{\infty ; 1},\left\|c_{0}\right\|_{\infty},\left\|c_{i j}\right\|_{\infty ; 1}$, and $G$ such that

$$
K_{t}(g ; h) \geq a t^{-d / 2} e^{-b\left|g h^{-1}\right|^{2} / t} e^{-\omega t}
$$

for all $g, h \in G$ and $t>0$. If $G=\mathbf{R}^{d}$ the constants $a, b$, and $\omega$ can be taken to be independent of $\left\|c_{i j}\right\|_{\infty ; 1}$.

(Here, $a, b$ are not necessarily the same $a, b$ as above. For economy of notation $a$ and $b$ will be used to denote different constants throughout the rest of the section.)

By the remark after Theorem 2.1, we may and will assume that $G$ is connected through the rest of this section. (Many of the lemmas in this section are wrong or meaningless, without this assumption.)

The proof of Theorem 4.1 involves five steps, some of which have independent interest. We begin with an outline of the five steps:

The first step is a covariance estimate. Define the gradient $\nabla \varphi$ for $\varphi$ in any appropriate function space on $G$ as the vector valued function

$$
\nabla \varphi(g)=\left(\left(A_{i} \varphi\right)(g)\right)_{i=1}^{d}
$$

and the Laplacian $\nabla^{2}$ by

$$
\nabla^{2} \varphi(g)=\sum_{i=1}^{d} A_{i}^{2} \varphi(g) .
$$

The covariance estimate alluded to above is the following generalization of Poincaré's inequality (in the case that $G=\mathbf{R}$ and $e^{-\alpha}$ has compact support, this is Lemma 3 in [Mos]): 
Lemma 4.2. Let $\alpha$ be a real twice continuously differentiable function on $G$ with the properties

$$
\begin{gathered}
\int_{G} d \hat{g} e^{-\alpha(g)}=1, \\
\lim _{|g| \rightarrow \infty}\left\{|\nabla \alpha(g)|^{2}-4 \nabla^{2} \alpha(g)\right\}=+\infty,
\end{gathered}
$$

and

$$
\lim _{|g| \rightarrow \infty}|\nabla \alpha(g)|^{2}=+\infty
$$

Let $\mu$ be the probability measure defined through $d \mu(g)=e^{-\alpha(g)} d \hat{g}$. It follows that there exists a constant $c>0$ depending only on $\alpha$ such that

$$
\mu\left(|\nabla f|^{2}\right) \geq c\left(\mu\left(f^{2}\right)-\mu(f)^{2}\right)
$$

for all real differentiable functions $f \in L_{2}(G ; d \mu)$. Equivalently,

$$
\int_{G} d \mu(g) \int_{G} d \mu(h)(f(g)-f(h))^{2} \leq c^{-1} \int_{G} d \mu(g)|\nabla f(g)|^{2} .
$$

This lemma is proved in $\S 4.1$ via a characterization of compact subsets of $L_{2}(G ; d \hat{g})$. It is then used to establish a lower bound on the relative entropy of $e^{-\alpha(g)} d \hat{g}$ with respect to $K_{t}(g ; h) d \hat{g}$,

$$
S\left(e^{-\alpha} \mid K_{t}\right)=-\int_{G} d \hat{g} e^{-\alpha(g)}\left(\log e^{-\alpha(g)}-\log K_{t}(g ; h)\right) .
$$

This relative entropy is nonpositive (and negative if $K_{t} \neq e^{-\alpha}$ ) by the following standard argument. We have $-x \log x \leq 1-x$ for $x>0$; thus,

$$
-(x / y) \log (x / y) \leq 1-x / y
$$

for $x, y>0$, and multiplying by $y$ :

$$
-x(\log x-\log y) \leq y-x .
$$

Thus, if $\rho_{1}$ and $\rho_{2}$ are probability densities,

$$
\begin{aligned}
S\left(\rho_{1} \mid \rho_{2}\right) & =-\int_{G} d \hat{g} \rho_{1}(g)\left(\log \left(\rho_{1}(g)\right)-\log \left(\rho_{2}(g)\right)\right) \\
& \leq \int_{G} d \hat{g}\left(\rho_{2}(g)-\rho_{1}(g)\right)=0 .
\end{aligned}
$$

As usual, we define the entropy of a probability density by

$$
S(\rho)=-\int_{G} d \hat{g} \rho(g) \log (\rho(g)) .
$$

In particular, $S(\rho)>-\infty$ if $\rho$ is bounded above.

Lemma 4.3. Let $\alpha$ be as in Lemma 4.2, i.e.,

1. $\int_{G} d \hat{g} e^{-\alpha(g)}=1$ and

2. $\lim _{|g| \rightarrow \infty}\left\{|\nabla \alpha(g)|^{2}-4 \nabla^{2} \alpha(g)\right\}=+\infty$ and $\lim _{|g| \rightarrow \infty}|\nabla \alpha(g)|^{2}=+\infty$. 


\section{Assume in addition}

3. $g \rightarrow \alpha(g)$ is bounded below, $\alpha(g) \geq-r$,

4. $\int_{G} d \hat{g} e^{-\alpha(g)}|\nabla \alpha(g)|^{2}<+\infty$,

5. $\int_{G} d \hat{g} e^{-\alpha(g)}|g|<+\infty$.

It follows that there exists a constant $b_{t}$ only depending on $\alpha, \lambda_{m}, \lambda_{M},\left\|c_{i}\right\|_{\infty ; 1}$, $\left\|c_{0}\right\|_{\infty},\left\|c_{i j}\right\|_{\infty ; 1}$, and $G$, such that

$$
\begin{gathered}
\int_{G} d \hat{g} e^{-\alpha(g)} \log K_{t}(g ; h) \geq-b_{t}, \\
\int_{G} d \hat{g} e^{-\alpha(g)} \log K_{t}(h ; g) \geq-b_{t}
\end{gathered}
$$

for $|h|<1$. (Then $S\left(e^{-\alpha(g)} \mid K_{t}(g ; h)\right)$ and $S\left(e^{-\alpha(g)} \mid K_{t}(h, g)\right.$ ) are likewise bounded below. The functions $g \rightarrow K_{t}(g ; h)$ and $g \rightarrow K_{t}(h ; g)$ are not necessarily probability distributions (the latter is so if $c_{0}=0$, the former if $c_{0}=0$, $\left.c_{i}=0\right)$.) The function $t \rightarrow b_{t}$ can be chosen to be uniformly bounded on compact subsets of $\langle 0,+\infty\rangle$ and to be increasing in $\left\|c_{i}\right\|_{\infty ; 1},\left\|c_{0}\right\|_{\infty}, \lambda_{M}$, and decreasing in $\lambda_{m}$. The constant $b_{t}$ is independent of $\left\|c_{i j}\right\|_{\infty ; 1}$ if $G$ is unimodular.

A typical function $\alpha$ satisfying the requirements of Lemma 4.3 would be a smoothing of the function $g \rightarrow|g|^{2}$.

This lemma is proved in $\S 4.2$.

The third small step is to bound $K_{t}(g ; h)$ below when $\left|g h^{-1}\right| \leq 1$ :

Lemma 4.4. It follows that $K_{t}(g ; h) \geq e^{-r-2 b_{t / 2}}$ for $\left|g h^{-1}\right| \leq 1$, where $b_{t}$ and $r$ are as in Lemma 4.3.

Proof. One has

$$
\begin{aligned}
K_{t}(g ; h) & =\int_{G} d \hat{k} K_{t / 2}(g ; k) K_{t / 2}(k ; h) \\
& \geq e^{-r} \int_{G} d \hat{k} e^{-\alpha(k)} K_{t / 2}(g ; k) K_{t / 2}(k ; h),
\end{aligned}
$$

where we used $\alpha(k) \geq-r$. But, since $\int_{G} d \hat{k} e^{-\alpha(k)}=1$ and log is a concave function, we deduce

$$
\begin{aligned}
\log K_{t}(g ; h) & \geq-r+\int_{G} d \hat{k} e^{-\alpha(k)} \log \left(K_{t / 2}(g ; k) K_{t / 2}(k ; h)\right) \\
& =-r+\int_{G} d \hat{k} e^{-\alpha(k)} \log K_{t / 2}(g ; k)+\int_{G} d \hat{k} e^{-\alpha(k)} \log K_{t / 2}(k ; h) \\
& \geq-r-2 b_{t / 2}
\end{aligned}
$$

for $|g| \leq 1,|h| \leq 1$ by Lemma 4.3. Thus, $K_{t}(g ; h) \geq e^{-r-2 b_{t / 2}}$ for $|g| \leq 1$, $|h| \leq 1$. To obtain the general estimate we note that if we conjugate the original operator

$$
C^{(p)}=-\sum_{i, j=1}^{d} A_{i} c_{i j} A_{j}+\sum_{i=1}^{d} c_{i} A_{i}+c_{0}
$$


with right translation, we obtain an operator of the same form, but the new coefficient functions $c_{i j}, c_{i}, c_{0}$ are right translates of the old ones. Thus, this operation does not change $\lambda_{m}, \lambda_{M},\left\|c_{i}\right\|_{\infty ; 1}$, and $\left\|c_{0}\right\|_{\infty}$. The kernel of the new operator has the form $(t, g, h) \rightarrow K_{t}(g k ; h k)$, where $K$ is the old kernel and $k$ is the right translate. Thus, applying the reasoning above, we obtain

$$
K_{t}(g k ; k h) \geq e^{-p-2 b_{t / 2}}
$$

if $|g| \leq 1,|h| \leq 1$ and for all $k \in G$. Applying this with $g$ replaced by $g h^{-1}$, $h$ by $e$, and $k$ by $h$ gives Lemma 4.4.

The fourth step is to use Lemma 4.4 in the special case $G=\mathbf{R}^{d}$ and a scaling argument to establish:

Lemma 4.5. There is a constant $a>0$ depending only on $\lambda_{m}, \lambda_{M},\left\|c_{i}\right\|_{\infty ; 1}$, $\left\|c_{0}\right\|_{1}$, and $G$, and a constant $\delta>0$ depending on these constants and $\left\|c_{i j}\right\|_{\infty ; 1}$ such that

$$
K_{t}(g ; h) \geq a t^{-d / 2}
$$

whenever $0<t<\delta$ and $\left|g h^{-1}\right| \leq \sqrt{t}$. If $G=\mathbf{R}^{d}$ we can take $\delta=1$.

At this point we remark that even though we use Lemma 4.4 only in the special case of $G=\mathbf{R}^{d}$, it does not seem that we can directly use existing results in [FaS and Nas] since these depend on $C^{(p)}$ being a pure second-order operator $\left(c_{i}=0, c_{0}=0\right)$. Another approach, used in [Var, pp. 369-370], is to combine the upper estimate with the parabolic Harnack inequality [Mos, Theorem 2; FaS, Theorem 5.4; or Bon, Théorème 7.1], but again all of these authors impose special conditions on $C^{(p)}$ which are not always fulfilled in our case. A hyperbolic Harnack inequality of the form

$$
\sup _{\left|g h^{-1}\right| \leq t^{1 / 2}} K_{t}(g ; h) \leq \gamma \inf _{\left|g h^{-1}\right| \leq t^{1 / 2}} K_{2 t}(g ; h)
$$

for $0<t \leq 1$, where $\gamma$ depends only on $\lambda_{m}, \lambda_{M},\left\|c_{i}\right\|_{\infty ; 1},\left\|c_{0}\right\|_{\infty}$, would imply Lemma 4.5 by Varapoulos' argument. Note conversely that our upper and lower estimates imply Harnack's inequality in the above form.

The proof of Theorem 4.1 from Lemma 4.5 is now exactly as the proof of Theorem 2.1 from Lemma 2.9 in [ABR]. Roughly, with $(g, t) \in G \times \mathbf{R}_{+}$given, one picks the smallest integer $k$ such that

$$
4 \frac{(|g| / k)^{2}}{t / k} \leq 1, \quad t / k \leq \delta,
$$

and chooses a sequence $g_{0}=e, g_{1}, \ldots, g_{k}=g$ such that

$$
\left|g_{i}^{-1} g_{i+1}\right| \leq|g| / k \text {. }
$$

One then uses the convolution formula

$$
\begin{aligned}
& K_{t}(g ; e)=\int_{G} d \hat{h}_{1} \cdots \int_{G} d \hat{h}_{k-1} K_{t / k}\left(g ; h_{k-1}\right) K_{t / k}\left(h_{k-1} ; h_{k-2}\right) \\
& \cdots K_{t / k}\left(h_{2} ; h_{1}\right) K_{t / k}\left(h_{1} ; e\right)
\end{aligned}
$$


and the estimate in Lemma 4.5, and restricts the above integration to $h_{i}$ lying in a ball of small radius around $g_{i}$. This gives an estimate of the form

$$
K_{t}(g ; e) \geq a^{k}\left(c(16 k)^{-d / 2}\right) k^{d / 2} t^{-d / 2} \text {. }
$$

Using $k-1 \leq 4|g|^{2} / t+t / \delta$, one now derives Theorem 4.1.

4.1 The covariance estimate. In this section we prove Lemma 4.2. To this end we need the following version of the Fréchet-Kolmogorov theorem [Yos].

Lemma 4.2.1. A subset $K$ of $L_{p}(G ; d g), 1 \leq p<\infty$, is strongly precompact if, and only if, the following three conditions are satisfied:

1. $\sup _{\varphi \in K}\|\varphi\|_{p}<+\infty$.

2. $\lim _{g \rightarrow e} \sup _{\varphi \in K}\|L(g) \varphi-\varphi\|_{p}=0$ and $\lim _{g \rightarrow e} \sup _{\varphi \in K}\|R(g) \varphi-\varphi\|_{p}=0$, where $R$ is the right regular representation.

3. $\lim _{a \rightarrow \infty} \sup _{\varphi \in K} \int_{|g|>a}|\varphi(g)|^{p} d g=0$.

It does not matter in condition 2 which definition of $R$ we use, i.e., $(R(g) \varphi)(h)=\varphi(h g)$ or the isometric definition $(R(g) \varphi)(h)=\varphi(h g) \Delta(g)^{1 / p}$. We will use the first definition in the following.

This theorem is proved for $G=\mathbf{R}$ in [Yos, X.1], and we may repeat the proof almost word for word: If $K$ is precompact, there exists for any $\varepsilon>0$ a finite set $\left\{\varphi_{1}, \ldots, \varphi_{n}\right\}$ of functions in $L_{p}(G ; d g)$ of compact support (each $\varphi$ may even be taken to be a finite linear combination of characteristic functions) such that each $\varphi \in K$ can be approximated within $\varepsilon$ in $L_{p}$ by one of the functions $\varphi_{i}$. But if $K$ is replaced by the finite set $\left\{\varphi_{1}, \ldots, \varphi_{n}\right\}$ in conditions 1,2 , and 3 , the resulting conditions are trivially satisfied, and then they are satisfied for $K$ by approximation.

Conversely, if $K$ satisfies conditions 1,2 , and 3, we may prove that $K$ is precompact by regularizing the elements in $K$ and applying the Ascoli-Arzelà theorem. We must show that for any $\varepsilon>0$ there exists a finite subset $\left\{\varphi_{1}, \ldots, \varphi_{n}\right\}$ in $K$ such that each $\varphi$ in $K$ has $L_{p}$-distance less than $\varepsilon$ to this set. First note that by condition 3 we may assume that all functions in $K$ are supported on a common compact set $\Omega$. Next, for any compact neighborhood $B$ of $e$ with characteristic function $\mathscr{X}$ and volume $|B|=\int_{B} d g$, introduce the right regularizations

$$
R_{B} \varphi=|B|^{-1} \int_{B} d g R(g) \varphi
$$

Then

$$
\begin{aligned}
\left\|R_{B} \varphi-\varphi\right\|_{P} & =\left\||B|^{-1} \int_{B} d g(R(g) \varphi-\varphi)\right\|_{p} \\
& =\left(\left.\left.\int_{G} d h\left|\int_{B} d g\right| B\right|^{-1}(\varphi(h g)-\varphi(h))\right|^{p}\right)^{1 / p} .
\end{aligned}
$$


But

$$
\begin{aligned}
\left.\left|\int_{B} d g\right| B\right|^{-1}(\varphi(h g)-\varphi(h)) \mid & \leq\left(\int_{B} d g|\varphi(h g)-\varphi(h)|^{p}\right)^{1 / p}\left(\int_{B} d g|B|^{-q}\right)^{1 / q} \\
& =|B|^{(1-q) / q}\left(\int_{B} d g|\varphi(h g)-\varphi(h)|^{p}\right)^{1 / p} \\
& =|B|^{-(1 / p)}\left(\int_{B} d g|\varphi(h g)-\varphi(h)|^{p}\right)^{1 / p}
\end{aligned}
$$

by Hölder's inequality. Thus,

$$
\begin{aligned}
\left\|R_{B} \varphi-\varphi\right\|_{p} & \leq\left(|B|^{-1} \int_{G} d h \int_{B} d g|\varphi(h g)-\varphi(h)|^{p}\right)^{1 / p} \\
& =\left(|B|^{-1} \int_{B} d g \int_{G} d h|\varphi(h g)-\varphi(h)|^{p}\right)^{1 / p} \\
& =\left(|B|^{-1} \int_{B} d g\|R(g) \varphi-\varphi\|_{p}^{p}\right)^{1 / p}
\end{aligned}
$$

by Fubini's theorem. But the last expression tends to zero, uniformly for $\varphi$ in $K$, as the diameter of $B$ tends to zero, by the last half of condition 2 . Thus we may replace $K$ by $R_{B} K$. But now for fixed $B$ we will argue that $R_{B} K$ is a uniformly bounded equicontinuous family of functions. Since all these functions are supported on the precompact set $\Omega B \subseteq G$, it then follows from Ascoli-Arzelà's theorem that these functions form a precompact subset of $C(\overline{\Omega B})$ in supremum norm, and since again $\overline{\Omega B}$ has finite measure, the $L_{p^{-}}$norm of these functions is dominated by the $L_{\infty}$-norm, and hence we finally deduce that $K$ is a precompact subset of $L_{p}(G ; d g)$. The equicontinuity of the set $R_{B} K$ follows from the estimate

$$
\begin{aligned}
\left|\left(R_{B} \varphi\right)\left(h^{-1} g\right)-\left(R_{B} \varphi\right)(g)\right| & \leq|B|^{-1} \int_{B} d k\left|\varphi\left(h^{-1} g k\right)-\varphi(g k)\right| \\
& \leq|B|^{-(1 / p)}\left(\int_{B} d k\left|\varphi\left(h^{-1} g k\right)-\varphi(g k)\right|^{p}\right)^{1 / p} \\
& \leq|B|^{-(1 / p)}\|L(h) \varphi-\varphi\|_{p}
\end{aligned}
$$

which tends to zero as $h \rightarrow e$, uniformly in $\varphi \in K$ and $g \in G$. Thus $R_{B} K$ is equicontinuous. Similarly, $\left|R_{B} \varphi(g)\right| \leq|B|^{-(1 / p)}\|\varphi\|_{p}$, so the family $R_{B} K$ is uniformly bounded. This ends the proof of Lemma 4.2.1.

To continue the proof of Lemma 4.2, define a positive bilinear form on $L_{2}\left(G ; e^{-\alpha(g)} d \hat{g}\right)$ by

$$
\begin{aligned}
a(\varphi, \psi) & =\int_{G} d \hat{g} e^{-\alpha(g)} \overline{\nabla \varphi(g)} \nabla \psi(g) \\
& =\sum_{i} \int_{G} d \hat{g} e^{-\alpha(g)} \overline{A_{i} \varphi(g)} A_{i} \psi(g)=\sum_{i}\left\langle A_{i} \varphi, e^{-\alpha} A_{i} \psi\right\rangle,
\end{aligned}
$$


where $\langle$,$\rangle denotes the inner product on L^{2}(G ; d \hat{g})$. We deduce that

$$
a(\varphi, \varphi)=\sum_{i}\left\|e^{-\alpha / 2} A_{i} e^{\alpha / 2} e^{-\alpha / 2} \varphi\right\|_{\hat{2}}^{2}=\sum_{i}\left\|\left(A_{i}+\frac{1}{2} \partial_{i} \alpha\right) e^{-\alpha / 2} \varphi\right\|_{\hat{2}}^{2},
$$

where $\left(\partial_{i} \alpha\right)(g)=\left.\frac{d}{d t} \alpha\left(e^{t a_{i}} g\right)\right|_{t=0}$. Now, the unitary map $\varphi \rightarrow e^{-(\alpha / 2)} \varphi$ from $L_{2}\left(G ; e^{-\alpha} d \hat{g}\right)$ into $L_{2}(G ; d \hat{g})$ transforms the bilinear form $a$ into a positive bilinear form $A$ given by

$$
A(\varphi, \varphi)=\sum_{i=1}^{d}\left\|\left(A_{i}+\frac{1}{2} \partial_{i} \alpha\right) \varphi\right\|_{\hat{2}}^{2} .
$$

Now, again use the relation $\left\langle A_{i} \varphi, \psi\right\rangle=-\left\langle\varphi,\left(A_{i}-\beta_{i} I\right) \psi\right\rangle$, where $\beta_{i}=\left(A_{i} \Delta\right)(e)$, to deduce

$$
\begin{aligned}
A(\varphi, \varphi)= & -\sum_{i=1}^{d}\left\langle\varphi,\left(A_{i}-\beta_{i} I\right) A_{i} \varphi\right\rangle-\frac{1}{2} \sum_{i=1}^{d}\left\langle\varphi,\left(A_{i}-\beta_{i} I\right)\left(\left(\partial_{i} \alpha\right) \varphi\right)\right\rangle \\
& +\frac{1}{2} \sum_{i=1}^{d}\left\langle\varphi,\left(\partial_{i} \alpha\right) A_{i} \varphi\right\rangle+\frac{1}{4} \sum_{i=1}^{d}\left\langle\varphi,\left(\partial_{i} \alpha\right)^{2} \varphi\right\rangle \\
= & \langle\nabla \varphi, \nabla \varphi\rangle-\frac{1}{2} \sum_{i=1}^{d}\left\langle\varphi,\left(\partial_{i}^{2} \alpha\right) \varphi\right\rangle \\
& +\frac{1}{2} \sum_{i=1}^{d}\left\langle\varphi, \beta_{i}\left(\partial_{i} \alpha\right) \varphi\right\rangle+\frac{1}{4} \sum_{i=1}^{d}\left\langle\varphi,\left(\partial_{i} \alpha\right)^{2} \varphi\right\rangle \\
= & \langle\nabla \varphi, \nabla \varphi\rangle+\left\langle\alpha,\left\{\frac{1}{4}(\nabla \alpha)^{2}+\frac{1}{2} \beta \nabla \alpha-\frac{1}{2} \nabla^{2} \alpha\right\} \varphi\right\rangle .
\end{aligned}
$$

By standard results [Kat, VI, $\S \S 1-2], A$ is a closable bilinear form, and its closure defines a positive self-adjoint operator $H$ which is an extension of

$$
-(\nabla-\beta) \nabla+\frac{1}{4}(\nabla \alpha)^{2}+\frac{1}{2}(\beta \nabla \alpha)-\frac{1}{2}\left(\nabla^{2} \alpha\right) .
$$

Lemma 4.2.2. The operator $H$ has compact resolvent, and $H$ has 0 as an eigenvalue with corresponding eigenvector $e^{-\alpha(g) / 2}$ unique to a scalar factor.

Proof. To show that $H$ has compact resolvent, it suffices to show that the set

$$
K=\left\{\varphi \in L_{2}(G ; d \hat{g}) ; A(\varphi, \varphi)+\lambda(\varphi, \varphi) \leq 1\right\}
$$

is compact in $L_{2}(G ; d \hat{g})$ for some $\lambda>0$. Choose $\lambda$ so large that

$$
\frac{1}{4}(\nabla \alpha)^{2}(g)+\frac{1}{2}(\beta \nabla \alpha)-\frac{1}{2}\left(\nabla^{2} \alpha\right)(g)+\lambda \geq 1
$$

for all $g$. This is possible by the second condition on $\alpha$. Thus, if $\varphi \in K$ we have

(i) $\langle\varphi, \varphi\rangle \leq 1$.

(ii) $\langle\nabla \varphi, \nabla \varphi\rangle \leq 1$.

(iii) $\left\langle\varphi,\left(\frac{1}{4}(\nabla \alpha)^{2}+\frac{1}{2}(\beta \nabla \alpha)-\frac{1}{2}\left(\nabla^{2} \alpha\right)+\lambda\right) \varphi\right\rangle \leq 1$. 
We now apply Lemma 4.2.1 with $p=2$. (Actually we need Lemma 4.2.1 with left Haar measure replaced by right but the proof is identical.) Clearly (1) follows from (i) and (3) follows from (iii) since

$$
\sup _{\varphi \in K} \int_{|g| \geq a}|\varphi(g)|^{2} d \hat{g} \leq\left(\inf _{|g| \geq a}\left(\frac{1}{4}(\nabla \alpha(g))^{2}+\frac{1}{2}(\beta \nabla \alpha)(g)-\frac{1}{2}\left(\nabla^{2} \alpha\right)(g)+\lambda\right)\right)^{-1}
$$

and the expression to the right tends to zero as $a$ tends to infinity. Finally we show that (2) follows from (ii). From (ii) it follows that $\left\|\sum_{i} c_{i} A_{i} \varphi\right\|_{\hat{2}} \leq 1$ when $\sum_{i} c_{i}^{2} \leq 1$ and $\varphi \in K$. Thus, as $\|L(g) \varphi\|_{\hat{2}}=\Delta(g)^{-1 / 2}\|\varphi\|_{\hat{2}}$,

$$
\begin{aligned}
\left\|L\left(e^{-t \sum_{i} c_{i} a_{i}}\right) \varphi-\varphi\right\|_{\hat{2}} & =\left\|\int_{0}^{1} d s L\left(e^{-s t \sum_{i} c_{i} a_{i}}\right) t \sum_{i} c_{i} d L\left(a_{i}\right) \varphi\right\|_{\hat{2}} \\
& \leq|t|\left(\Delta\left(e^{t / 2 \sum_{i} c_{i} a_{i}}\right) \vee 1\right) .
\end{aligned}
$$

Hence

$$
\lim _{g \rightarrow e} \sup _{\varphi \in K}\|L(g) \varphi-\varphi\|_{\hat{2}}=0 .
$$

In order to show the corresponding estimate for right translations, we first note that by (3) we may approximate the elements $\varphi \in K$ uniformly by elements $\varphi^{\prime}$ in $L_{2}(G ; d g)$ with supports in a compact subset $\Omega \subseteq G$. We can do this by first setting $\varphi^{\prime}(g)=\varphi(g) \chi_{\Omega}(g)$, and by using the already derived equicontinuity under left translations and regularizing the $\varphi^{\prime}$ 's by a fixed positive $C^{\infty}$-function $\psi$ with small support near the origin and integral 1 . The functions

$$
\varphi^{\prime}=\int_{G} d g \psi(g) L(g) \varphi,
$$

i.e.,

$$
\begin{aligned}
\varphi^{\prime}(g) & =\int_{G} d h \psi(h) \varphi\left(h^{-1} g\right) \chi_{\Omega}\left(h^{-1} g\right) \\
& =\int_{G} d h \Delta(h)^{-1} \psi\left(g h^{-1}\right) \varphi(h) \chi_{\Omega}(h),
\end{aligned}
$$

are still close to $\varphi$ in $L_{2}$-norm, uniformly. All the functions $\varphi^{\prime}$ have support in the precompact set $\operatorname{supp}(\psi) \cdot \Omega$ and $\nabla \varphi^{\prime}=(\nabla \psi) *\left(\varphi \chi_{\Omega}\right)$ are equibounded in $L_{2}$-norm. Now there exist constants $a$ and $b$, only depending on the structure of the Lie algebra of $G$, such that

$$
\left|\left(d R\left(a_{i}\right) \chi\right)(g)\right| \leq a e^{b|g|} \sum_{i=1}^{d}\left|\left(d L\left(a_{i}\right) \chi\right)(g)\right|
$$

for all differentiable functions $\chi$ on $G$ (see [BGJR, Lemma 3.4]). Since all the functions $\varphi^{\prime}$ have support in a common compact, it follows that also the right-derivatives of $\varphi^{\prime}$ are equibounded in $L_{2}$-norm. One now deduces as for left-translations that

$$
\lim _{|g| \rightarrow 0} \sup _{\varphi \in K}\left\|R(g) \varphi^{\prime}-\varphi^{\prime}\right\|_{\hat{2}}=0
$$


and, using the estimate

$$
\begin{aligned}
\|R(g) \varphi-\varphi\|_{\hat{2}} & \leq\left\|R(g)\left(\varphi-\varphi^{\prime}\right)\right\|_{\hat{2}}+\left\|R(g) \varphi^{\prime}-\varphi^{\prime}\right\|_{\hat{2}}+\left\|\varphi-\varphi^{\prime}\right\|_{\hat{2}} \\
& \leq 2\left\|\varphi-\varphi^{\prime}\right\|_{\hat{2}}+\left\|R(g) \varphi^{\prime}-\varphi^{\prime}\right\|_{\hat{2}},
\end{aligned}
$$

one deduces

$$
\lim _{|g| \rightarrow 0} \sup _{\varphi \in K}\|R(g) \varphi-\varphi\|_{\hat{2}}=0 .
$$

It follows from Lemma 4.2.1 that $K$ is compact, and hence $H$ has compact resolvent.

For the assertion on the zero eigenvalue of $H$, note that

$$
\left\|H^{1 / 2} e^{-\alpha / 2} \varphi\right\|_{\hat{2}}^{2}=\int_{G} d \hat{g} e^{-\alpha(g)}|\nabla \varphi(g)|^{2}
$$

by the computations before the lemma. We see that the right-hand side is zero if, and only if, $\nabla \varphi=0$, i.e., $\varphi$ is a constant (since $G$ is connected). This ends the proof of Lemma 4.2.2.

The proof of the covariance estimate in Lemma 4.2 is now immediate. As $H$ has compact resolvent there is a gap $c>0$ from 0 to the next smallest eigenvalue of $H$. Let $P_{0}$ be the projection onto the eigenspace of $H$ corresponding to 0 , i.e.,

$$
P_{0} \varphi=\left\langle e^{-\alpha / 2}, \varphi\right\rangle e^{-\alpha / 2}
$$

It follows from spectral theory that

$$
\begin{aligned}
\langle\varphi, H \varphi\rangle & =\left\langle\varphi, H\left(1-P_{0}\right) \varphi\right\rangle \geq c\left\langle\varphi,\left(1-P_{0}\right) \varphi\right\rangle \\
& =c\left(\langle\varphi, \varphi\rangle-\left\langle\varphi, e^{-\alpha / 2}\right\rangle\left\langle e^{-\alpha / 2}, \varphi\right\rangle\right) .
\end{aligned}
$$

Replacing $\varphi$ by $e^{-\alpha / 2} \varphi$ in this estimate one obtains

$$
a(\varphi, \varphi) \geq c\left(\left\langle e^{-\alpha / 2} \varphi, e^{-\alpha / 2} \varphi\right\rangle-\left|\left\langle e^{-\alpha / 2}, e^{-\alpha / 2} \varphi\right\rangle\right|^{2}\right)
$$

or

$$
\int_{G} d \hat{g} e^{-\alpha(g)}|(\nabla \varphi)(g)|^{2} \geq c\left[\int_{G} d \hat{g} e^{-\alpha(g)}|\varphi(g)|^{2}-\left|\int_{G} d \hat{g} e^{-\alpha(g)} \varphi(g)\right|^{2}\right],
$$

which is the sought after covariance estimate in Lemma 4.2.

4.2 The relative entropy estimate. In this section we prove Lemma 4.3. As remarked after Theorem 2.1 , if $K_{t}(g ; h)$ is the semigroup kernel associated with the operator

$$
-\sum_{i, j=1}^{d} A_{i} c_{i j} A_{j}+\sum_{i=1}^{d} c_{i} A_{i}+c_{0}
$$

then $K_{t}^{*}(g ; h)=K_{t}(h ; g) \Delta\left(g^{-1} h\right)$ is the semigroup kernel associated with the adjoint

$$
-\sum_{i, j=1}^{d} A_{j} c_{i j} A_{i}-\sum_{i=1}^{d} c_{i} A_{i}+\left(c_{0}-\sum_{i=1}^{d} A_{i}\left(c_{i}\right)\right)
$$


of this operator. The relevant constants associated with the latter operator are $\lambda_{m}, \lambda_{M},\left\|c_{i}\right\|_{\infty, 1}$, and $\left\|c_{0}-\sum_{i=1}^{d} A_{i}\left(c_{i}\right)\right\|_{\infty} \leq\left\|c_{0}\right\|_{\infty}+\sum_{i=1}^{d}\left\|c_{i}\right\|_{\infty ; 1}$. Thus, once we can show

$$
G(t) \equiv \int_{G} d \hat{g} e^{-\alpha(g)} \log K_{t}(g ; h) \geq-b_{t}\left(\lambda_{m}, \lambda_{M},\left\|c_{i}\right\|_{\infty ; 1},\left\|c_{0}\right\|_{\infty}\right),
$$

it will follow that

$$
\begin{aligned}
\int_{G} d \hat{g} e^{-\alpha(g)} \log \left(K_{t}(h ; g) \Delta(g)^{-1} \Delta(h)\right) \\
\quad \geq-b_{t}\left(\lambda_{m}, \lambda_{M},\left\|c_{i}\right\|_{\infty ; 1},\left\|c_{0}\right\|_{\infty}+\sum_{i=1}^{d}\left\|c_{i}\right\|_{\infty ; 1}\right) .
\end{aligned}
$$

But as $g \rightarrow \log \Delta(g)^{-1}$ is a continuous additive character we have an estimate $\left|\log \Delta(g)^{-1}\right| \leq a|g|$, where $a$ only depends on $G$. Thus, using (5) and the fact that $\Delta(h)$ is bounded away from zero when $|h|$ is bounded, the second estimate in Lemma 4.3 follows from the first (with $b_{t}$ suitably modified). Hence it suffices to prove $(*)$ above.

We insert a remark on relative entropy: Since $\alpha \geq-r$, we have

$$
S\left(e^{-\alpha}\right)=-\int_{G} d \hat{g} e^{-\alpha(g)}(-\alpha(g)) \geq-r .
$$

Also $S\left(e^{-\alpha} \mid K_{t}(\cdot ; h)\right)=S\left(e^{-\alpha}\right)+G(t)$. Next, using

$$
S\left(\rho_{1} \mid \rho_{2}\right) \leq \int_{G} d \hat{g}\left(\rho_{2}(g)-\rho_{1}(g)\right)
$$

which is valid whenever $\rho_{1}, \rho_{2}$ are positive functions, we deduce

$$
S\left(e^{-\alpha}\right)+G(t) \leq \int_{G} d \hat{g}\left(K_{t}(g ; h)-e^{-\alpha(g)}\right)=\int_{G} d \hat{g} K_{t}(g ; h)-1 .
$$

But now it follows from the upper estimate (3.2) in Theorem 3.1 that

$$
\int_{G} d \hat{g} K_{t}(g ; h) \leq c e^{\omega t}
$$

for a suitable constant $c$, where we used that the volume of the ball around $h$ with radius $x$ grows at most exponentially when $x \rightarrow \infty$, and is $O\left(x^{d}\right)$ when $x \rightarrow 0$. Hence $G(t) \leq c e^{\omega t}-1+r$, where $c, \omega$ depend only on $\lambda_{m}, \lambda_{M},\left\|c_{i}\right\|_{\infty, 1},\left\|c_{0}\right\|_{\infty}$.

To obtain the lower estimate on $G(t)$, we note that $G$ is differentiable by 
Theorem 2.1 and

$$
\begin{aligned}
\frac{d G}{d t}= & \int_{G} d \hat{g} e^{-\alpha(g)} K_{t}(g ; h)^{-1} \frac{d}{d t}\left(K_{t}(g ; h)\right) \\
= & -\int_{G} d \hat{g} e^{-\alpha(g)} K_{t}(g ; h)^{-1}\left(C^{\infty} K_{t}\right)(g ; h) \\
= & \sum_{i, j=1}^{d} \int_{G} d \hat{g} e^{-\alpha(g)}\left(A_{i} c_{i j} A_{j}\right)\left(K_{t}(g ; h)\right) K_{t}(g ; h)^{-1} \\
& -\sum_{i=1}^{d} \int_{G} d \hat{g} e^{-\alpha(g)}\left(c_{i} A_{i}\right)\left(K_{t}(g ; h)\right) K_{t}(g ; h)^{-1} \\
& -\int_{G} d \hat{g} e^{-\alpha(g)} c_{0} .
\end{aligned}
$$

Now we again use that the adjoint of $A_{i}$ with respect to $d \hat{g}$ is $-A_{i}+\beta_{i} 1$, where $\beta_{i}=A_{i}(\Delta)(e)$. If this is used in the first addend above we obtain expressions like

$$
\begin{aligned}
\left(-A_{i}\right. & \left.+\beta_{i} 1\right)\left(e^{-\alpha(g)} K_{t}(g ; h)^{-1}\right) \\
= & e^{-\alpha(g)} K_{t}(g ; h)^{-2} A_{i}\left(K_{t}(g ; h)\right) \\
& +e^{-\alpha(g)} A_{i}(\alpha(g)) K_{t}(g ; h)^{-1}+e^{-\alpha(g)} \beta_{i} K_{t}(g ; h)^{-1}
\end{aligned}
$$

Hence

$$
\begin{aligned}
\frac{d G}{d t}= & \sum_{i, j=1}^{d} \int_{G} d \hat{g} e^{-\alpha(g)} c_{i j}(g) A_{i}\left(\log K_{t}(g ; h)\right) A_{j}\left(\log K_{t}(g ; h)\right) \\
& +\sum_{i, j=1}^{d} \int_{G} d \hat{g} e^{-\alpha(g)} c_{i j}(g) A_{i}(\alpha(g)) A_{j}\left(\log K_{t}(g ; h)\right) \\
& +\sum_{i, j=1}^{d} \int_{G} d \hat{g} e^{-\alpha(g)} c_{i j}(g) \beta_{i} A_{j}\left(\log K_{t}(g ; h)\right) \\
& -\sum_{j=1}^{d} \int_{G} d \hat{g} e^{-\alpha(g)} c_{j}(g) A_{j}\left(\log K_{t}(g ; h)\right)-\int_{G} d \hat{g} e^{-\alpha(g)} c_{0}(g) \\
\geq & \lambda_{m} \sum_{i=1}^{d} \int_{G} d \hat{g} e^{-\alpha(g)}\left|A_{i}\left(\log K_{t}(g ; h)\right)\right|^{2} \\
& +\sum_{i, j=1}^{d} \int_{G} d \hat{g} e^{-\alpha(g)}\left\{c_{i j}(g) A_{i}(\alpha(g))+c_{i j}(g) \beta_{i}-\delta_{i j} c_{i}(g)\right\} \\
& \times A_{j}\left(\log K_{t}(g ; h)\right) \\
& -\int_{G}^{d \hat{g} e^{-\alpha(g)} c_{0}(g) .}
\end{aligned}
$$


We now estimate the last two terms. The integrand in

$$
\sum_{i, j=1}^{d} \int_{G} d \hat{g} e^{-\alpha(g)} A_{i}(\alpha(g)) c_{i j}(g) A_{j}\left(\log K_{t}(g ; h)\right)
$$

has the form $(u, C v)$, where $C$ is the positive matrix $\left(C_{i j}(g)\right)$. As $C$ is positive we have

$$
((\sqrt{\varepsilon} u \pm 1 /(2 \sqrt{\varepsilon}) v), C(\sqrt{\varepsilon} u \pm 1 /(2 \sqrt{\varepsilon}) v)) \geq 0
$$

i.e.,

$$
\begin{aligned}
|(u, C v)| & \leq \varepsilon(u, C u)+(1 / 4 \varepsilon)(v, C v) \\
& \leq \varepsilon \lambda_{M}(u, u)+1 /(4 \varepsilon) \lambda_{M}(v, v)
\end{aligned}
$$

Thus,

$$
\begin{gathered}
\left|\sum_{i, j=1}^{d} \int_{G} d \hat{g} e^{-\alpha(g)} A_{i}(\alpha(g)) c_{i j}(g) A_{j}\left(\log K_{t}(g ; h)\right)\right| \\
\leq \varepsilon \lambda_{M} \sum_{i=1}^{d} \int_{G} d \hat{g} e^{-\alpha(g)}\left|A_{i}\left(\log K_{t}(g ; h)\right)\right|^{2} \\
\quad+1 /(4 \varepsilon) \lambda_{M} \sum_{i=1}^{d} \int_{G} d \hat{g} e^{-\alpha(g)}\left|A_{i}(\alpha(g))\right|^{2}
\end{gathered}
$$

Similarly,

$$
\begin{aligned}
& \left|\sum_{i, j=1}^{d} \int_{G} d \hat{g} e^{-\alpha(g)} \beta_{i} c_{i j}(g) A_{j}\left(\log K_{t}(g ; h)\right)\right| \\
& \quad \leq \varepsilon \lambda_{M} \sum_{i=1}^{d} \int_{G} d \hat{g} e^{-\alpha(g)}\left|A_{i}\left(\log K_{t}(g ; h)\right)\right|^{2}+1 /(4 \varepsilon) \lambda_{M} \sum_{i=1}^{d}\left|\beta_{i}\right|^{2} .
\end{aligned}
$$

The elementary estimate

$$
\left|c_{i}(g) A_{i}\left(\log K_{t}(g ; h)\right)\right| \leq \varepsilon \lambda_{M}\left|A_{i}\left(\log K_{t}(g ; h)\right)\right|^{2}+1 /\left(4 \varepsilon \lambda_{M}\right)\left|c_{i}(g)\right|^{2}
$$

implies

$$
\begin{aligned}
& \left|\sum_{i=1}^{d} \int_{G} d \hat{g} e^{-\alpha(g)} c_{i}(g) A_{i}\left(\log K_{t}(g ; h)\right)\right| \\
& \quad \leq \varepsilon \lambda_{M} \sum_{i=1}^{d} \int_{G} d \hat{g} e^{-\alpha(g)}\left|A_{i}\left(\log K_{t}(g ; h)\right)\right|^{2}+1 /\left(4 \varepsilon \lambda_{M}\right) \sum_{i=1}^{d}\left\|c_{i}\right\|_{\infty}^{2} .
\end{aligned}
$$

Finally,

$$
\left|\int_{G} d \hat{g} e^{-\alpha(g)} c_{0}(g)\right| \leq\left\|c_{0}\right\|_{\infty} .
$$


Inserting these estimates in the estimate for $d G / d t$ we obtain

$$
\begin{aligned}
\frac{d G}{d t} \geq & \left(\lambda_{m}-3 \varepsilon \lambda_{M}\right) \sum_{i=1}^{d} \int_{G} d \hat{g} e^{-\alpha(g)}\left|A_{i}\left(\log K_{t}(g ; h)\right)\right|^{2} \\
& -1 /(4 \varepsilon) \lambda_{M} \sum_{i=1}^{d}\left[\int_{G} d \hat{g} e^{-\alpha(g)}\left|A_{i}(\alpha(g))\right|^{2}+\left|\beta_{i}\right|^{2}+1 /\left(\lambda_{M}^{2}\right)\left\|c_{i}\right\|_{\infty}^{2}\right]-\left\|c_{0}\right\|_{\infty} .
\end{aligned}
$$

Now, fixing $\varepsilon>0$ once and for all such that $\lambda_{m}-3 \varepsilon \lambda_{M}>0$ and using assumption (4) on $\alpha$ we obtain the estimate

$$
\frac{d G}{d t} \geq a \int_{G} d \hat{g} e^{-\alpha(g)}\left|\nabla\left(\log K_{t}(g ; h)\right)\right|^{2}-b,
$$

where $a, b$ are finite positive constants depending on $\lambda_{m}, \lambda_{M},\left\|c_{i}\right\|_{\infty},\left\|c_{0}\right\|_{\infty}$, $\alpha$, and $G$, but nothing more.

By Lemma 4.2 there exists a constant $c$, depending only on $\alpha$, such that

$$
\begin{aligned}
\int_{G} d \hat{g} e^{-\alpha(g)}\left|\nabla\left(\log K_{t}(g ; h)\right)\right|^{2} \\
\quad \geq c\left(\int_{G} d \hat{g} e^{-\alpha(g)}\left(\log K_{t}(g ; h)\right)^{2}-\left[\int_{G} d \hat{g} e^{-\alpha(g)} \log K_{t}(g ; h)\right]^{2}\right) \\
\quad=c\left(\int_{G} d \hat{g} e^{-\alpha(g)}\left(\log \left(K_{t}(g ; h)\right)-G(t)\right)^{2}\right) .
\end{aligned}
$$

Hence

$$
\frac{d G}{d t} \geq a c\left(\int_{G} d \hat{g} e^{-\alpha(g)}\left(\log K_{t}(g ; h)-G(t)\right)^{2}\right)-b .
$$

Now, for $t>0$ given, put $c_{t}=\sup \left\{K_{s}(g ; h) ; t / 2 \leq s \leq t, g, h \in G\right\}$. Then $c_{t}$ is a finite constant depending only on $\lambda_{m}, \lambda_{M},\left\|c_{i}\right\|_{\infty ; 1},\left\|c_{0}\right\|_{\infty}$, and $t$ by the upper estimate in Theorem 3.1. Since the function $u \rightarrow(\log u-G(t))^{2} / u$ is nonincreasing on $\left[e^{2+G(t)}, \infty\right\rangle$ we obtain the estimate

$$
\frac{d G}{d s} \geq-b+a c\left(\left(\log c_{t}-G(s)\right)^{2} / c_{t}\right) \cdot \int_{B_{s}} d \hat{g} e^{-\alpha(g)} K_{s}(g ; h)
$$

for $t / 2 \leq s \leq t$, where $B_{s}=\left\{g ; K_{t}(g ; h) \geq e^{2+G(s)}\right\}$. But if $I$ is the latter integral we have

$$
\begin{aligned}
I & \geq \int_{G} d \hat{g} e^{-\alpha(g)} K_{s}(g ; h)-e^{2+G(s)} \\
& \geq \int_{\{g ;|g| \leq R\}} d \hat{g} e^{-\alpha(g)} K_{s}(g ; h)-e^{2+G(s)}
\end{aligned}
$$

for any $R>0$. If $E(R)=\inf \left\{e^{-\alpha(g)} ;|g| \leq R\right\}$, we further estimate

$$
\begin{aligned}
I & \geq E(R) \int_{\{g ;|g| \leq R\}} d \hat{g} K_{s}(g ; h)-e^{2+G(s)} \\
& =E(R)\left(\int_{G} d \hat{g} K_{s}(g ; h)-\int_{\{g ;|g| \geq R\}} d \hat{g} K_{s}(g ; h)\right)-e^{2+G(s)} .
\end{aligned}
$$


Now we use the relation

$$
K_{t}(h ; g)=\Delta(g) K_{t}^{*}(g ; h) \Delta(h)^{-1}
$$

which implies that $(g, h) \rightarrow K_{t}(h ; g)$ is the heat kernel of the operator

$$
\Delta C^{*} \Delta^{-1}=\Delta\left(-\sum_{i, j=1}^{d} A_{i} c_{i j} A_{j}-\sum_{i=1}^{d} c_{i} A_{i}+c_{0}-\sum_{i=1}^{d} A_{i}\left(c_{i}\right)\right) \Delta^{-1} .
$$

Now

$$
\Delta A_{i} \Delta^{-1}=A_{i}+A_{i}\left(\Delta^{-1}\right)(e)=A_{i}-\beta_{i},
$$

where again $\beta_{i}=A_{i}(\Delta)(e)$. Thus the operator in question is

$$
\begin{aligned}
& -\sum_{i, j=1}^{d}\left(A_{i}-\beta_{i}\right) c_{i j}\left(A_{j}-\beta_{j}\right)-\sum_{i=1}^{d} c_{i}\left(A_{i}-\beta_{i}\right)+c_{0}-\sum_{i=1}^{d} A_{i} c_{i} \\
& =\sum_{i, j=1}^{d}\left(A_{i}-\beta_{i}\right) c_{i j} \beta_{j}+\sum_{i=1}^{d} c_{i} \beta_{i}+c_{0}-\sum_{i=1}^{d} A_{i}\left(c_{i}\right)+R \\
& =\sum_{i, j=1}^{d} A_{i}\left(c_{i j}\right) \beta_{j}-\sum_{i, j=1}^{d} \beta_{i} c_{i j} \beta_{j}+\sum_{i=1}^{d} c_{i} \beta_{i}+c_{0}-\sum_{i=1}^{d} A_{i}\left(c_{i}\right)+R,
\end{aligned}
$$

where $R$ indicates additional terms having a differentiation to the right. It now follows from Theorem 2.1 that $\int_{G} d \hat{g} K_{s}(g ; h) \geq e^{-s \omega_{M}}$, where

$$
\begin{aligned}
\omega_{M} & =\sup \left\{\sum_{i, j=1}^{d} A_{i}\left(c_{i j}\right) \beta_{j}-\sum_{i, j=1}^{d} \beta_{i} c_{i j} \beta_{j}+\sum_{i=1}^{d} c_{i} \beta_{i}+c_{0}-\sum_{i=1}^{d} A_{i}\left(c_{i}\right)\right\} \\
& \leq c\left(\sum_{i, j=1}^{d}\left\|c_{i j}\right\|_{\infty ; 1}+\sum_{i=1}^{d}\left\|c_{i}\right\|_{\infty ; 1}+\left\|c_{0}\right\|_{\infty}\right) ;
\end{aligned}
$$

here $c$ is a constant depending only on $G$. Note that if $G$ is unimodular, then $\beta_{i}=0, i=1, \ldots, d$, and we obtain the simpler estimate

$$
\omega_{M} \leq\left\|c_{0}\right\|_{\infty}+\sum_{i=1}^{d}\left\|c_{i}\right\|_{\infty ; 1} .
$$

This is the only point in the proof where $\left\|c_{i j}\right\|_{\infty ; 1}$ enters.

Now, it follows from the upper estimate on $K_{t}$ in Theorem 3.1 that we can choose an $R>0$ such that

$$
\int_{\{g ;|g|>R\}} d \hat{g} K_{s}(g ; h) \leq e^{-t \omega_{M} / 2}
$$

whenever $t / 2 \leq s \leq t$ and $|h| \leq 1$, where $R$ depends only on $\lambda_{m}, \lambda_{M},\left\|c_{i}\right\|_{\infty ; 1}$, $\left\|c_{0}\right\|_{\infty}$. Choosing $R$ this large, we estimate

$$
I \geq E(R) e^{-t \omega_{M}} / 2-e^{2+G(s)}
$$


and hence

$$
\frac{d G}{d s} \geq-b+a c\left(\left(\log c_{t}-G(s)\right)^{2} / c_{t}\right) \cdot\left(E(R) e^{-t \omega_{M}} / 2-e^{2+G(s)}\right)
$$

whenever $t / 2 \leq s \leq t$.

We know from an earlier estimate that $d G / d s \geq-b$; thus, $s \rightarrow G(s)+b s$ is a nondecreasing function of $t$, i.e.,

$$
G(s)+b s \leq G(t)+b t
$$

or

$$
G(s) \leq G(t)+b(t-s) \leq G(t)+b t / 2
$$

for $t / 2 \leq s \leq t$. Hence

$$
\frac{d G}{d s} \geq-b+a c\left(\left(\log c_{t}-G(s)\right)^{2} / c_{t}\right) \cdot\left(E(R) e^{-t \omega_{M}} / 2-e^{2+G(t)+b t / 2}\right)
$$

for $t / 2 \leq s \leq t$. From this estimate we see that there exist new positive constants $a$ and $c$ depending only on the usual parameters, such that if $G(t)<$ $-a$, then $d G / d s \geq c G(s)^{2}$ for $t / 2 \leq s \leq t$. But this means that $-\frac{d}{d s}(1 / G(s)) \geq$ $c$. Integrating from $t / 2$ to $t$, we obtain

$$
1 / G(t / 2)-1 / G(t) \geq c t / 2 \text {. }
$$

We may choose $a$ so large that $G(t / 2) \leq-a+b t / 2<0$ and then $G(t) \geq$ $-(2 / c t)$. Thus, we deduce in any case that $G(t) \geq \min \{-a,-2 / c t\}$.

This terminates the proof of Lemma 4.3 except for the uniform boundedness of $b_{t}$ on intervals of the form $\left[\delta, \delta^{-1}\right]$, where $0<\delta<1$. But this follows by combining the pointwise bound of $G(\delta)$ with the fact that $G(t)+b t$ is a nondecreasing function of $t$ for a suitable constant $b$ depending only on $\lambda_{m}, \lambda_{M},\left\|c_{i}\right\|_{\infty ; 1},\left\|c_{0}\right\|_{\infty}$.

4.3 The scaling argument. We know from Lemma 4.4 that

$$
K_{t}(g ; h) \geq a\left(t, \lambda_{m}, \lambda_{M},\left\|c_{i}\right\|_{\infty ; 1},\left\|c_{0}\right\|_{\infty},\left\|c_{i j}\right\|_{\infty ; 1}\right)>0
$$

whenever $\left|g h^{-1}\right|<1$ and $t>0$, where $t \rightarrow a(t)$ is uniformly bounded away from zero on compact intervals in $\langle 0, \infty\rangle$. In this section we will prove Lemma 4.5 , i.e., we show that $a$ can be taken to have the form

$$
\begin{aligned}
& a\left(t, \lambda_{m}, \lambda_{M},\left\|c_{i}\right\|_{\infty ; 1},\left\|c_{0}\right\|_{\infty},\left\|c_{i j}\right\|_{\infty ; 1}\right) \\
& =a\left(\lambda_{m}, \lambda_{M},\left\|c_{i}\right\|_{\infty ; 1},\left\|c_{0}\right\|_{\infty},\left\|c_{i j}\right\|_{\infty ; 1}\right) t^{-d / 2}
\end{aligned}
$$

for $\left|g h^{-1}\right|<\sqrt{t}$ and $0<t<\delta$, where $\delta>0$ only depends on $G$.

We start with the case $G=\mathbf{R}^{d}$. Since $G$ is unimodular we have the estimate

$$
K_{1}(g ; h) \geq a\left(\lambda_{m}, \lambda_{M},\left\|c_{i}\right\|_{\infty ; 1},\left\|c_{0}\right\|_{\infty}\right)>0
$$

Now, introduce a positive scale $\mu$ and rescale the variables: $t \rightarrow \mu^{2} t, g \rightarrow \mu g$. Define

$$
C^{\mu}=-\sum_{i, j=1}^{d} A_{i} c_{i j}(\mu g) A_{j}+\mu \sum_{i=1}^{d} c_{i}(\mu g) A_{i}+\mu^{2} c_{0}(\mu g)
$$


Then, if $\varphi$ is a solution of $\partial / \partial t \varphi(g ; t)+C \varphi(g ; t)=0$, we have

$$
C^{\mu}\left(\varphi\left(\mu g ; \mu^{2} t\right)\right)=\mu^{2}(C \varphi)\left(\mu g ; \mu^{2} t\right)
$$

and

$$
\frac{\partial}{\partial t} \varphi\left(\mu g ; \mu^{2} t\right)=\mu^{2}\left(\frac{\partial \varphi}{\partial t}\right)\left(\mu g ; \mu^{2} t\right) .
$$

Thus $\psi(g ; t)=\varphi\left(\mu g ; \mu^{2} t\right)$ is a solution of $\left(\frac{\partial}{\partial t}+C^{\mu}\right) \psi=0$ and the heat kernel $K^{\mu}$ associated to $C^{\mu}$ must be related to $K$ by

$$
K_{t}^{\mu}(g ; h)=c(\mu) K_{\mu^{2} t}(\mu g ; \mu h) ;
$$

where $c(\mu)$ is a constant. Since

$$
K_{0}(\mu g ; \mu h)=\delta(\mu(g-h))=\left(1 / \mu^{d}\right) \delta(g-h)
$$

and $K_{0}^{\mu}(g ; h)=\delta(g-h)$, we obtain $c(\mu)=\mu^{d}$ and hence

$$
K_{t}^{\mu}(g ; h)=\mu^{d} K_{\mu^{2} t}(\mu g ; \mu h) .
$$

But now the lower and upper ellipticity constants associated to $C^{\mu}$ are exactly the same as those of $C$, whilst the $\left\|c_{i}\right\|_{\infty ; 1}$ and $\left\|c_{0}\right\|_{\infty}$ constants are at most $\mu^{2}$ times those of $C$. Thus we have

$$
\begin{aligned}
K_{1}^{\mu}(g ; h) & \geq a\left(\lambda_{m}, \lambda_{M}, \mu^{2}\left\|c_{i}\right\|_{\infty ; 1}, \mu^{2}\left\|c_{0}\right\|_{\infty}\right) \\
& \geq a\left(\lambda_{m}, \lambda_{M},\left\|c_{i}\right\|_{\infty ; 1},\left\|c_{0}\right\|_{\infty}\right)
\end{aligned}
$$

provided $\mu \leq 1$. (We may assume that $a$ is increasing in $\lambda_{m}$ and decreasing in the three other variables, since a decrease in the minimum permissible value of $\lambda_{m}$ and an increase in the maximum permissible value of the other variables corresponds to an extension of the permitted operators $C$.) Thus $\mu^{d} K_{\mu^{2}}(\mu g ; \mu h) \geq a$ provided $0<\mu<1$ and $|g-h| \leq 1$. But setting $\mu^{2}=t$ this means

$$
K_{t}(g ; h) \geq t^{-d / 2} a\left(\lambda_{m}, \lambda_{M},\left\|c_{i}\right\|_{\infty ; 1},\left\|c_{0}\right\|_{\infty}\right)
$$

whenever $|g-h|<\sqrt{t}$ and $0<t<1$. This establishes a strong version of Lemma 4.5 for $G=\mathbf{R}^{d}$, and $\delta$ can take any value.

For a general $G$ we cannot use a global scaling argument as above, but the exponential map allows us to scale locally: Let $\Omega$ be a compact neighborhood of $e$ in $G$ and $\hat{\Omega}$ a compact neighborhood of 0 in $\mathbf{R}^{d}$ such that the exponential map

$$
x \in \hat{\Omega} \rightarrow \exp \left(\sum_{i=1}^{d} x_{i} a_{i}\right) \in \Omega
$$

is a diffeomorphism. This map transforms the operator $C$ on $\Omega$ into an operator $\hat{C}$ on $\hat{\Omega}$ of the form

$$
\hat{C}=-\sum_{i, j=1}^{d} \frac{\partial}{\partial x_{i}} \hat{c}_{i j}(x) \frac{\partial}{\partial x_{i}}+\sum_{i=1}^{d} \hat{c}_{i}(x) \frac{\partial}{\partial x_{i}}+\hat{c}_{0}(x),
$$


where the coefficients $\hat{c}_{i j}, \hat{c}_{i}$, and $\hat{c}_{0}$ can be obtained from $c_{i j}, c_{i}$, and $c_{0}$ by suitable analytic transformations. More precisely, the operators $A_{i}$ transform into operators of the form

$$
\hat{A}_{i}=\sum_{j=1}^{d} \alpha_{i j}(x) \frac{\partial}{\partial x_{j}},
$$

where $\alpha_{i j}$ are analytic functions depending only on $G$ and $\alpha_{i j}(0)=\delta_{i j}$. Thus, $C$ transforms into

$$
\begin{aligned}
\hat{C}= & -\sum_{i, j=1}^{d} \sum_{k, l=1}^{d} \alpha_{i k}(x) \frac{\partial}{\partial x_{k}} c_{i j}(x) \alpha_{j l}(x) \frac{\partial}{\partial x_{l}} \\
& +\sum_{i=1}^{d} \sum_{k=1}^{d} c_{i}(x) \alpha_{i k}(x) \frac{\partial}{\partial x_{k}}+c_{0}(x),
\end{aligned}
$$

where we have written $c_{i j}\left(e^{a \cdot x}\right)=c_{i j}(x)$, etc. Since

$$
\alpha_{i k} \frac{\partial}{\partial x_{k}}=\frac{\partial}{\partial x_{k}} \alpha_{i k}-\frac{\partial}{\partial x_{k}}\left(\alpha_{i k}\right),
$$

we deduce that

$$
\begin{aligned}
\hat{c}_{i j}(x) & =\sum_{k, l=1}^{d} \alpha_{k i}(x) c_{k l}(x) \alpha_{l j}(x), \\
\hat{c}_{i}(x) & =\sum_{k=1}^{d} \alpha_{k i}(x) c_{k}(x)+\sum_{j, k, l=1}^{d} \frac{\partial}{\partial x_{k}}\left(\alpha_{l k}(x)\right) c_{l j}(x) \alpha_{j i}(x), \\
\hat{c}_{0}(x) & =c_{0}(x) .
\end{aligned}
$$

In particular, $\hat{c}_{i j}(0)=c_{i j}(e)$ and by choosing $\hat{\Omega}$ sufficiently small we may ensure that $\hat{\lambda}_{m}>\frac{1}{2} \lambda_{m}, \hat{\lambda}_{M}<2 \lambda_{M}$, and

$$
\left\|\hat{c}_{i}\right\|_{\infty ; 1}<c\left(\sum_{i}\left\|c_{i}\right\|_{\infty ; 1}+\sum_{i, j}\left\|c_{i j}\right\|_{\infty ; 1}\right), \quad\left\|\hat{c}_{0}\right\|_{\infty}=\left\|c_{0}\right\|_{\infty},
$$

where $c$ is a constant depending only on $G$, and the sup-norms of the capped variables are taken over $\hat{\Omega}$.

We then extend $\hat{c}_{i j}, \hat{c}_{i}$, and $\hat{c}_{0}$ from $\hat{\Omega}$ to all of $\mathbf{R}^{d}$ in such a way that all the coefficients have the right differentiability properties and the above estimates are valid in all of $\mathbf{R}^{d}$.

Let $F_{t}(x ; y)$ be the heat kernel associated to $\hat{C}$, now defined on all of $\mathbf{R}^{d}$ by the expression above. Let $P_{t}(x ; y)$ be the restriction of the kernel $K_{t}(g ; h)$ to $\Omega \times \Omega$, transported to $\hat{\Omega} \times \hat{\Omega}$ with the exponential map:

$$
P_{t}(x ; y)=K_{t}(\exp (x \cdot a) ; \exp (y \cdot a)) .
$$


Then both $F_{t}$ and $P_{t}$ satisfy the equations

$$
\left(\frac{\partial}{\partial t}+\hat{C}\right) F_{t}(x ; y)=0, \quad\left(\frac{\partial}{\partial t}+\hat{C}\right) P_{t}(x ; y)=0
$$

inside $\hat{\Omega}$, where $\hat{C}$ acts on the $x$-variable, with the initial condition

$$
F_{0}(x ; y)=\delta(x-y), \quad P_{0}(x ; y)=\delta(x-y)
$$

(we assume that the metric system on $G$ is chosen so that the exponential map maps the $\delta$-distribution on 0 into the $\delta$-distribution on $e$ ). Thus, if $G_{t}(x ; y)=F_{t}(x ; y)-P_{t}(x ; y)$, then $G$ satisfies the equation

$$
\left(\frac{\partial}{\partial t}+\hat{C}\right) G_{t}(x ; y)=0
$$

inside $\hat{\Omega}$, with the initial condition $G_{0}(x ; y)=0$.

Now let $\hat{\Omega}_{0}$ be a compact neighborhood of 0 in $\mathbf{R}^{d}$ such that $\hat{\Omega}_{0} \subseteq \hat{\Omega}$ and $\hat{\Omega}_{0}$ has a positive distance to the complement of $\hat{\Omega}$. If $y \in \hat{\Omega}_{0}$, then it follows from the upper estimates of $F_{t}(x ; y)$ and $P_{t}(x ; y)$ that $G_{t}$ satisfies a bound

$$
\left|G_{t}(x ; y)\right| \leq b\left(\lambda_{m},\left\|c_{i}\right\|_{\infty ; 1},\left\|c_{0}\right\|_{\infty}\right)
$$

for all $x \in \partial \hat{\Omega}, y \in \hat{\Omega}_{0}$, and $0 \leq t \leq 1$. It follows from the maximum principle for parabolic equations that $\left|G_{t}(x, y)\right| \leq b e^{\left\|c_{0}\right\|_{\infty} t}$ for all $x \in \hat{\Omega}, y \in \hat{\Omega}_{0}$, and $0 \leq t \leq 1$. Now, as we have already shown that

$$
F_{t}(x ; y) \geq t^{-d / 2} a\left(\lambda_{m} / 2,2 \lambda_{M}, 2\left\|c_{i}\right\|_{\infty ; 1}, 2\left\|c_{0}\right\|_{\infty}\right)
$$

for all $0 \leq t \leq 1$ and all $x, y \in \mathbf{R}^{d}$ with $|x-y|<\sqrt{t}$, it follows that

$$
P_{t}(x ; y) \geq t^{-d / 2} a-b e^{\left\|c_{0}\right\|_{\infty}}
$$

for all $x, y \in \hat{\Omega}_{0}$ such that $|x-y| \leq \sqrt{t}$ and all $0<t \leq 1$. Lemma 4.5 follows immediately from this estimate.

\section{REFERENCES}

[ABR] W. Arendt, C. J. K. Batty, and D. W. Robinson, Positive semigroups generated by elliptic operators on Lie groups, J. Operator Theory 23 (1990), 369-408.

[BGJR] O. Bratteli, F. M. Goodman, P. E. T. Jørgensen, and D. W. Robinson, The heat semigroup and integrability of Lie algebras, J. Funct. Anal. 79 (1988) 351-397.

[Bon] J. M. Bony, Principe du maximum, unicité du problème de Cauchy et inégalité de Harnack pour les opérateurs elliptiques dégénérés, Ann. Inst. Fourier (Grenoble) 19 (1969) 277-304.

[BR] O. Bratteli and D. W. Robinson, Operator algebras and quantum statistical mechanics. I, 2nd ed., Springer-Verlag, 1987.

[CFKS] H. L. Cycon, R. G. Froese, W. Kirsch, and B. Simon, Schrödinger operators, SpringerVerlag, 1987.

[CKS] E. A. Carlin, A. Kusuoko, and D. W. Stroock, Upper bounds for symmetric Markov transition functions, Ann. Inst. H. Poincaré Probab. Statist. 2 (1987), 245-287.

[Dav] E. B. Davies, Explicit constants for Gaussian upper bounds on heat kernels, Amer. J. Math. 109 (1987) 319-333. 
[FaS] E. B. Fabes and D. W. Stroock, A new proof of Moser's Harnack inequality using the old ideas of Nash, Arch. Rational Mech. Anal. 96 (1986) 327-338.

[Gảr] L. Gárding, Vecteurs analytiques dans des representations des groupes de Lie, Bull. Soc. Math. France 88 (1960), 73-93.

[Kat] T. Kato, Perturbation theory for linear operators, Springer-Verlag, 1966.

[Mos] J. Moser, A Harnack inequality for parabolic differential equations, Comm. Pure Appl. Math. 17 (1964) 101-134.

[Nas] J. Nash, Continuity of solutions of parabolic and elliptic equations, Amer. J. Math. 80 (1958) 931-954.

[Rob] D. W. Robinson, Elliptic differential operators on Lie groups, J. Funct. Anal. (to appear).

[Ste1] E. Stein, Interpolation of linear operators, Trans. Amer. Math. Soc. 83 (1956) 482-492.

[Ste2] _ Topics in harmonic analysis, Ann. of Math. Studies, no. 63, Princeton Univ. Press, Princeton, N. J., 1970.

[Tre] F. Trèves, Topological vector spaces, distributions, and kernels, Academic Press, 1967.

[Var] N. Th. Varopoulos, Analysis on Lie groups, J. Funct. Anal. 76 (1988) 346-410.

[Yos] K. Yosida, Functional analysis, Springer-Verlag, 1974.

Department of Mathematics, Institute of Advanced Studies, Australian National University, CANberra, Australia 